\title{
Mergers of Binary Neutron Star Systems: A Multimessenger Revolution
}

\author{
Elena Pian * \\ INAF, Astrophysics and Space Science Observatory, Bologna, Italy
}

On August 17, 2017, less than two years after the direct detection of gravitational radiation from the merger of two $\sim 30 \mathrm{M}_{\odot}$ black holes, a binary neutron star merger was identified as the source of a gravitational wave signal of $\sim 100 \mathrm{~s}$ duration that occurred at less than 50 Mpc from Earth. A short gamma-ray burst was independently identified in the same sky area by the Fermi and INTEGRAL satellites for high energy astrophysics, which turned out to be associated with the gravitational event. Prompt follow-up observations at all wavelengths led first to the detection of an optical and infrared source located in the spheroidal Galaxy NGC4993 and, with a delay of $\sim 10$ days, to the detection of radio and $X$-ray signals. This article revisits these observations and focusses on the early optical/ infrared source, which was thermal in nature and powered by the radioactive decay of the

OPEN ACCESS

Edited by: Livio Lamia,

University of Catania, Italy

Reviewed by:

Toshitaka Kajino,

The University of Tokyo, Japan Fiorella Burgio, Ministry of Education, Universities and Research, Italy

*Correspondence: Elena Pian elena.pian@inaf.it

Specialty section: This article was submitted to Nuclear Physics, a section of the journal Frontiers in Astronomy and Space Sciences

Received: 23 September 2020 Accepted: 02 December 2020 Published: 25 January 2021

Citation:

Pian E (2021) Mergers of Binary Neutron Star Systems: A Multimessenger Revolution. Front. Astron. Space Sci. 7:609460. doi: 10.3389/fspas.2020.609460 unstable isotopes of elements synthesized via rapid neutron capture during the merger and in the phases immediately following it. The far-reaching consequences of this event for cosmic nucleosynthesis and for the history of heavy elements formation in the Universe are also illustrated.

Keywords: gamma-ray burst, gravitational waves, neutron star, nucleosynthesis, r-process, kilonova

\section{INTRODUCTION}

Although the birth of "multimessenger" astronomy dates back to the detection of the first solar neutrinos in the 1960s and was rejuvenated by the report of MeV neutrinos from SN 1987A in the Large Magellanic Cloud, the detection of gravitational radiation from the binary neutron star merger on August 17, 2017 (GW170817A), marks the transition to maturity of this approach to observational astrophysics, as it is expected to open an effective window into the study of astrophysical sources which is not limited to exceptionally close (the Sun) or rare (Galactic supernova) events. GW170817 is a textbook case for gravitational physics, because, with its accompanying short gamma-ray burst (GRB) and afterglow and its thermal aftermath "kilonova", it has epitomized the different epiphanies of the coalescence of a binary system of neutron stars and finally allowed us to unify them.

Owing its name to a typical peak luminosity of $\sim 10^{42} \mathrm{erg} \mathrm{s}^{-1}$, i.e., 1000 times larger than that of a typical nova outburst, kilonova is the characteristic optical and infrared source accompanying a binary neutron star merger due to the radioactive decay of the many unstable isotopes of large atomic weight elements synthesized via rapid neutron capture in the promptly formed dynamical ejecta and in the delayed postmerger ejecta. Its evolution, as well as that of the GRB afterglow, was recorded with exquisite detail, thanks to its closeness $(40 \mathrm{Mpc})$. The scope of this article is to review the electromagnetic multiwavelength observations of GW170817 with particular attention to the kilonova phenomenon. 
The outline of the review is as follows: Section 2 sets the context of binary systems of neutrons stars and describes the predicted outcomes of their coalescences; Section 3 presents the case of GW170817, the only so far confirmed example of double neutron star merger and the multiwavelength features of its electromagnetic counterpart (short GRB and kilonova); Section 4 focusses on the kilonova, elaborates on its observed optical and near-infrared light curves and spectra, draws the link with nucleosynthesis of heavy elements, and outlines the theoretical framework that is necessary to describe the kilonova properties and implications; Section 5 summarizes the results and provides an outlook of this line of research in the near future.

\section{BINARY NEUTRON STAR MERGERS}

Neutron stars are the endpoints of massive stars evolution and therefore ubiquitous in the Universe: on average, they represent about $0.1 \%$ of the total stellar content of a Galaxy. Since massive stars are mostly in binary systems (Sana et al., 2012), neutron star binaries should form readily, if the supernova explosion of either progenitor massive star does not disrupt the system (Renzo et al., 2019). Alternatively, binary neutron star systems can form dynamically in dense environments like stellar clusters (see Ye et al., 2020 and references therein). Binary systems composed by a neutron star and a black hole are also viable, but rare (Pfahl et al., 2005), which may account for the fact that none has so far been detected in our Galaxy.

The prototype binary neutron star system in our Galaxy is PSR $\mathrm{B} 1913+16$, where one member was detected as a pulsar in a radio survey carried out at the Arecibo Observatory (Hulse and Taylor, 1974), and the presence of its companion was inferred from the periodic changes in the observed pulsation period of $59 \mathrm{~ms}$ (Hulse and Taylor, 1975). Among various tests of strong general relativity enabled by the radio monitoring of this binary system, which earned the Nobel Prize for Physics to the discoverers in 1993, was the measurement of the shrinking of the binary system orbit, signaled by the secular decrease of the $7.75 \mathrm{~h}$ orbital period, that could be entirely attributed to energy loss via gravitational radiation (Taylor and Weisberg, 1982; Weisberg and Huang, 2016 and references therein).

With an orbital decay rate of $\dot{P}=-2.4 \times 10^{-12} \mathrm{~s} \mathrm{~s}^{-1}$, the merging time of the PSR B1913 + 16 system is $\sim 300$ Myr. Following the detection of PSR B1913 + 16, another dozen of binary neutron stars systems were detected in our Galaxy (e.g., Wolszczan, 1991; Burgay et al., 2003; Tauris et al., 2017; Martinez et al., 2017). Almost half of these have estimated merging times significantly shorter than a Hubble time. The campaigns conducted by the LIGO interferometers in Sep 2015-Jan 2016 (first observing run) and, together with Virgo, in Nov 2016-Aug 2017 (second observing run), the latter leading to the first detection of gravitational waves from a merging double neutron star system (see Section 3), constrained the local merger rate density to be $110-3,840 \mathrm{Gpc}^{-3} \mathrm{yr}^{-1}$ (Abbott et al., 2019). This is consistent with previous estimates (see, e.g., Burgay et al., 2003), and, under a series of assumptions, marginally consistent with independent estimates based on double neutron star system formation in the classical binary evolution scenario (Chruslinska et al., 2018). Ye et al. (2020) have estimated that the fraction of merging binary neutron stars that have formed dynamically in globular clusters is negligible. Under the assumption that the event detected by LIGO on April 25, 2019 , was produced by a binary neutron star coalescence, the local rate of neutron star mergers would be updated to $250-2810$ $\mathrm{Gpc}^{-3} \mathrm{yr}^{-1}$ (Abbott et al., 2020a).

The merger of a binary neutron star system has four predicted outcomes: (1) a gravitational wave signal that is mildly isotropic, with a stronger intensity in the polar direction than in the equatorial plane; (2) a relativistic outflow, which is highly anisotropic and can produce an observable high energy transient; (3) a thermal, radioactive source emitting most of its energy at ultraviolet, optical, and near-infrared wavelengths; and (4) a burst of MeV neutrinos (Eichler et al., 1989; Rosswog and Liebendörfer, 2003) following the formation of the central remnant and possibly of high-energy $(>\mathrm{GeV})$ neutrinos from hadronic interactions within the relativistic jet (Fang and Metzger, 2017; Kimura et al., 2018). While neutrinos are extremely elusive and detectable only from very small distances with present instrumentation (see Section 5), the first three observables have been now all detected, as detailed in the next three subsections.

\subsection{Gravitational Waves}

Coalescing binary systems of degenerate stars and stellar mass black holes are optimal candidates for the generation of gravitational waves detectable from ground-based interferometers as the strong gravity conditions lead to huge velocities and energy losses (Shapiro and Teukolsky, 1983), and the frequency of the emitted gravitational waves reaches several $\mathrm{kHz}$, where the sensitivity of the advanced LIGO, Virgo, and KAGRA interferometers is designed to be maximal (Abbott et al., 2018).

The time behavior of binary systems of compact stars consists of three phases: a first inspiral phase in a close orbit that shrinks as gravitational radiation of frequency proportional to the orbital frequency is emitted, a merger phase where a remnant compact body is produced as a result of the coalescence of the two stars, and a postmerger, or ringdown, phase where the remnant still emits gravitational radiation while settling to its new stable configuration. During the inspiral, the amplitude of the sinusoidal gravitational signal rapidly increases as the distance between the two bodies decreases and the frequency increases (chirp), while in the ringdown phase the signal is an exponentially damped sinusoid. This final phase may encode critical information on the equation of state of the newly formed remnant (a black hole or, in the case of light neutron stars, a massive neutron star or metastable supramassive neutron star). The mathematical tool that is used to describe this evolution is the waveform model that aims at reproducing the dynamics of the system through the application of post-Newtonian corrections of increasing order and at providing the essential parameters that can then be compared with the interferometric observations (Blanchet, 2014; Nakano et al., 2019). 
Since the amplitude of gravitational waves depends on the masses of the binary member stars, the signal will be louder and thus detectable from larger distances, for binary systems that involve black holes than those with neutron stars. The current horizon for binary neutron star merger detection with LIGO is $200 \mathrm{Mpc}$, and 25-30\% smaller with Virgo and KAGRA (Abbott et al., 2018). The dependence of the gravitational waves amplitude on the physical parameters of the system implies that gravitational wave sources are standard sirens (Schutz, 1986), provided account is taken of the correlation between the luminosity distance and the inclination of the orbital plane with respect to the line of sight (Nissanke et al., 2010; Abbott et al., 2016).

\subsection{Short Gamma-Ray Bursts}

GRBs, flashes of radiation of $100-1,000 \mathrm{keV}$ that outshine the entire Universe in this band, have durations between a fraction of a second and hundreds or even thousands of seconds. However, the duration distribution is bimodal, with a peak around $0.2 \mathrm{~s}$ (short or subsecond GRBs) and one around 20 s (long GRBs; Kouveliotou et al., 1993). This bimodality is reflected in the spectral hardness, which is on average larger in short GRBs, and in a physical difference between the two groups. While most long GRBs are associated with core-collapse supernovae (Galama et al., 1998; Woosley and Bloom, 2006; Levan et al., 2016), subsecond GRBs are produced by the merger of two neutron stars or a neutron star and a black hole, as long predicted based on circumstantial evidence (Blinnikov et al., 1984; Eichler et al., 1989; Fong et al., 2010; Berger et al., 2013; Tanvir et al., 2013) and then proven by the detection of GW170817 and of its high energy counterpart GRB170817A (Section 3). The observed relative ratio of long vs. short GRBs depends on the detector sensitivity and effective energy band (e.g., Burns et al., 2016). However, the duration overlap of the two populations is very large, so that the minimum of the distribution has to be regarded as a rather vaguely defined value (Bromberg et al., 2013).

About 140 short GRBs were localized so far to a precision that is better than 10 arc-minutes $^{1}$; of these, $\sim 100, \sim 40$, and $\sim 10$ have a detected afterglow in X-rays, optical, and radio wavelengths, respectively, and $\sim 30$ have measured redshifts (these range between $z=0.111$ and $z=2.211$, excluding the nearby GRB170817A, see Section 3.1.1, and GRB090426, $z=2.61$, whose identification as a short GRB is not robust, Antonelli et al., 2009). Short GRBs are located at projected distances of a fraction of, to several kiloparsecs from, the centers of their host galaxies, which are of both early and late type, reflecting the long time delay between the formation of the short GRB progenitor binary systems and their mergers (Berger, 2014).

According to the classical fireball model, both prompt event and multiwavelength afterglow of short GRBs are produced in a highly relativistic jet directed at a small angle with respect to the line of sight, whose aperture can be derived from the achromatic steepening (or "jet break") of the observed afterglow light curve

${ }^{1}$ http://www.mpe.mpg.de/jcg/grbgen.html
(Nakar, 2007). In principle, this could be used to reconstruct the collimation-corrected rate of short GRBs, to be compared with predictions of binary neutron star merger rates. However, these estimates proved to be very uncertain, owing to the difficulty of measuring accurately the jet breaks in short GRB afterglows (Wanajo et al., 2002; Fong et al., 2015; Jin et al., 2018; Lamb et al., 2019; Pandey et al., 2019).

\subsection{R-process Nucleosynthesis}

Elements heavier than iron cannot form via stellar nucleosynthesis, as no enough neutrons are available for the formation of nuclei and temperatures are not sufficiently high to overcome the repulsive Coulomb barrier that prevents acquisition of further baryons into nuclei (Burbidge et al., 1957). Supernovae (especially the thermonuclear ones) produce large amounts of iron via decay (through ${ }^{56} \mathrm{Co}$.) of radioactive ${ }^{56} \mathrm{Ni}$ synthesized in the explosion. Heavier nuclei form via four neutron capture processes (Thielemann et al., 2011), the dominant ones being slow and rapid neutron capture, in brief s- and r-process, respectively, where "slow" and "rapid" refer to the timescale of neutron accretion into the nucleus with respect to that of the competing process of $\beta^{-}$decay. In the s-process, neutron captures occur with timescales of hundreds to thousands of years, making $\beta^{-}$decay highly probable, while r-process neutron capture occurs on a timescale of $\sim 0.01 \mathrm{~s}$, leading to acquisition of many neutrons before $\beta^{-}$decay can set on. As a consequence, the s-process produces less unstable, longer-lived isotopes, close to the socalled valley of $\beta$-stability (the decay time of a radioactive nucleus correlates inversely with its number of neutrons), while the r-process produces the heaviest, neutron-richest, and most unstable isotopes of heavy nuclei, up to uranium (Sneden et al., 2008; Mennekens and Vanbeveren, 2014; Thielemann et al., 2017; Côté et al., 2018; Horowitz et al., 2019; Kajino et al., 2019; Cowan et al., 2020). Among both s-process and r-process elements, some are particularly stable owing to their larger binding energies per nucleon, which causes their abundances to be relatively higher than others. In the abundances distribution in the solar neighborhood, these are seen as maxima "peaks" centered around atomic numbers $Z=39$ (Sr-Y-Zr), 57 (Ba-La-Ce-Nd), and $82(\mathrm{~Pb})$ for the s-process and, correspondingly somewhat lower atomic numbers $Z=35$ (Se-Br$\mathrm{Kr}$ ), 53 (Te-I-Xe), and 78 ( Ir-Pt-Au) for the r-process (e.g., Cowan et al., 2020).

Both s-process and r-process naturally occur in environments that are adequately supplied with large neutron fluxes. For the s-process, these are eminently asymptotic giant branch stars, where neutron captures are driven by the ${ }^{13} \mathrm{C}(\alpha, n){ }^{16} \mathrm{O}$ and ${ }^{22} \mathrm{Ne}$ $(\alpha, n)^{25} \mathrm{Mg}$ reactions (Busso et al., 1999). The r-process requires much higher energy and neutron densities, which are only realized in most physically extreme environments. While it can be excluded that big-bang nucleosynthesis can accommodate heavy elements formation in any significant amount (Rauscher et al., 1994), there is currently no consensus on the relative amounts of nucleosynthetic yields in the prime r-process candidate sites: core-collapse supernovae and mergers of binary systems composed by neutron stars or a neutron star and a black hole. 
Core-collapse supernovae have been proposed starting many decades ago as sites of $r$-process nucleosynthesis through various mechanisms and in different parts of the explosion, including dynamical ejecta of prompt explosions of O-Ne-Mg cores (Hillebrandt et al., 1976; Wheeler et al., 1998; Wanajo et al., 2002); C + O layer of O-Ne-Mg-core supernovae (Ning et al., 2007); He-shell exposed to intense neutrino flux (Epstein et al., 1988; Banerjee et al., 2011); re-ejection of fallback material (Fryer et al., 2006); neutrino-driven wind from protoneutron stars (Takahashi et al., 1994; Woosley et al., 1994); and magnetohydrodynamic jets of rare core-collapse $\mathrm{SNe}$ (Nishimura et al., 2006; Winteler et al., 2012). Similarly old is the first proposal that the tidal disruption of neutron stars by black holes in close binaries (Lattimer and Schramm, 1974, 1976; Symbalisty and Schramm, 1982; Davies et al., 1994) and coalescences of binary neutron star systems (Eichler et al., 1989) could be at the origin of r-process nucleosynthesis. This should manifest as a thermal optical-infrared source of radioactive nature of much lower luminosity (a factor of 1,000) and shorter duration (rise time of a few days) than supernova (Li \& Paczyński, 1998).

The models for r-process elements production in core-collapse supernova all have problems inherent in their physics (mostly related to energy budget and neutron flux density). On the other hand, the binary compact star merger origin may fail to explain observed r-process element abundances in very low metallicities stars, i.e., at very early cosmological epochs, owing to the nonnegligible binary evolution times (see Cowan et al., 2020 for an accurate review of all arguments in favor and against either channel). While the event of August 17, 2017 (Section 3), has now provided incontrovertible evidence that binary neutron star mergers host r-process nucleosynthesis, the role of core-collapse supernovae cannot be dismissed although their relative contribution with respect to the binary compact star channel must be assessed (Ramirez-Ruiz et al., 2015; Ji et al., 2016; Shibagaki et al., 2016; Côté et al., 2019; Safarzadeh et al., 2019; Simonetti et al., 2019). It cannot be excluded that both "weak" and "strong" r-process nucleosyntheses take place, with the former occurring mainly in supernova and possibly failing to produce atoms up to the third peak of r-process elemental abundance distribution (Cowan et al., 2020). The hint that heavy elements may be produced in low-rate events with high yields (Sneden et al., 2008; Wallner et al., 2015; Macias and Ramirez-Ruiz, 2019) points to binary compact star mergers or very energetic (i.e., expansion velocities larger than $20,000 \mathrm{~km} \mathrm{~s}^{-1}$ ) corecollapse supernovae as progenitors, rather than regular core-collapse supernovae. Along these lines, it has been proposed that accretion disks of collapsars (the powerful core-collapse supernovae that accompany long GRBs, Woosley and Bloom, 2006) produce neutron-rich outflows that synthesize heavy r-process nuclei (Nakamura et al., 2013; Kajino et al., 2014; Nakamura et al., 2015). Siegel et al. (2019) calculated that collapsars may supply more than $80 \%$ of the r-process content and computed synthetic spectra for models of r-process-enriched supernovae corresponding to an MHD supernova and a collapsar disk outflow scenario.

Neutrons are tightly packed together in neutrons stars, but during coalescence of a binary neutron star system the tidal forces disrupt them and the released material forms promptly a disk-like rotating structure (dynamical ejecta, Rosswog et al., 1999; Shibata and Hotokezaka, 2019) where the neutron density rapidly drops to optimal values for r-process occurrence $\left(\sim 10^{24-32}\right.$ neutrons $\mathrm{cm}^{-3}$, Freiburghaus et al., 1999) and for copious formation of neutron-rich stable and unstable isotopes of large atomic number elements (Fernández and Metzger, 2016; Tanaka, 2016; Tanaka et al., 2018; Wollaeger et al., 2018; Metzger, 2019).

\section{THE BINARY NEUTRON STAR MERGER OF 17 AUGUST, 2017}

On August 17, 2017, the LIGO and Virgo interferometers detected for the first time a gravitational signal that corresponds to the final inspiral and coalescence of a binary neutron star system (Abbott et al., 2017a). The sky uncertainty area associated with the event was 28 square degrees, in principle too large for a uniform search for an electromagnetic counterpart with ground-based and orbiting telescopes. However, its small distance $\left(40_{-14}^{+8} \mathrm{Mpc}\right)$, estimated via the "standard siren" property of gravitational wave signals associated with binary neutron star mergers, suggested that the aftermath could be rather bright and motivated a large-scale campaign at all wavelengths from radio to very high energy gamma-rays, which was promptly and largely rewarded by success and then timely followed by a long and intensive monitoring (Abbott et al., 2017b; Abbott et al., 2017c), as described in Section 3.1. Searches of MeV-to-EeV neutrinos directionally coincident with the source using data from the Super-Kamiokande, ANTARES, IceCube, and Pierre Auger Observatories between $500 \mathrm{~s}$ before and 14 days after the merger returned no detections (Albert et al., 2017; Abe et al., 2018).

Based on the detection of electromagnetic radiation, Bauswein et al. (2017) have argued that the merger remnant may not be a black hole or at least the postmerger collapse to a black hole may be delayed. Since the postmerger phase "ring-down" signal of GW170817 was not detected (Abbott et al., 2017e), this hypothesis cannot be tested directly with gravitational data. Bauswein et al. (2017) also derived lower limits on the radii of the neutron stars.

Notably, while the gravitational data made it possible to set an upper limit on the tidal-deformability parameter of the binary neutron stars $(\tilde{\Lambda} \lesssim 800$, Abbott et al., 2017a), the optical observation of kilonova ejecta limited the same parameter from below ( $\tilde{\Lambda} \gtrsim 400$, Radice et al., 2018), based on the consideration that for smaller values of $\tilde{\Lambda}$ a long-lived remnant would not be favored, contradicting the result of Bauswein et al. (2017). The limits on the $\tilde{\Lambda}$ parameter constrain the neutron star radius to the range $11.8 \mathrm{~km}$ $\lesssim R_{1.5} \lesssim 13.1 \mathrm{~km}$, where $R_{1.5}$ refers to a $1.5 M_{\odot}$ neutron star (Burgio et al., 2018), and in turn confine the possible ensemble of viable equations of state (Annala et al., 2018; Lim and Holt, 2018), a fundamental, yet poorly known, descriptor of neutron star physics (Özel and Freire, 2016). Furthermore, by circumscribing the number of equations of state of the compact stars, their exploration can be brought beyond nucleonic matter and extended to scenarios of matter presenting a phase transition 
(Burgio et al., 2018; Most et al., 2018). The results on the tidal deformability of the neutron star progenitors of GW170817 and on the behavior of the remnant thus provide a brilliant confirmation of the added value of a multimessenger approach over separate observations of individual carriers of information.

\subsection{The Electromagnetic Counterpart of GW170817}

Independent of LIGO-Virgo detection of the gravitational wave signal, the Gamma-ray Burst Monitor (GBM) onboard the NASA Fermi satellite and the Anticoincidence Shield for the gamma-ray Spectrometer (SPI) of the International Gamma-Ray Astrophysics Laboratory (INTEGRAL) satellite were triggered by a faint short GRB (duration of $\sim 2 \mathrm{~s}$ ), named GRB170817A (Abbott et al., 2017b; Goldstein et al., 2017; Savchenko et al., 2017). This gamma-ray transient, whose large error box was compatible with that determined by LIGO-Virgo, lags the gravitational merger by $1.7 \mathrm{~s}$, a delay that may be dominated by the propagation time of the jet to the gamma-ray production site (Beniamini et al., 2020; see however Salafia et al., 2018). The preliminary estimate of the source distance provided a crucial constraint on the maximum distance of the Galaxy that could plausibly have hosted the merger, so that the searching strategy was based on targeting galaxies within a $\sim 50 \mathrm{Mpc}$ cosmic volume (see, e.g., Gehrels et al., 2016) with telescopes equipped with large (i.e., several square degrees) field-of-view cameras.

About 70 ground-based optical telescopes participated in the hunt and each of them adopted a different pointing sequence. This systematic approach enabled many groups to identify the optical counterpart candidate in a timely manner (with optical magnitude $V \simeq 17$ ), i.e., within $\sim 12 \mathrm{~h}$ of the merger (Arcavi et al., 2017; Lipunov et al., 2017; Soares-Santos et al., 2017; Valenti et al., 2017; Tominaga et al., 2018). Coulter et al. (2017) were the first to report a detection with the optical $1 \mathrm{~m}$ telescope Swope at Las Campanas Observatory. The optical source lies at 10 arc-seconds angular separation, corresponding to a projected distance of $\sim 2$ kpc, from the center of the spheroidal Galaxy NGC 4993 at 40 Mpc (Blanchard et al., 2017; Im et al., 2017; Levan et al., 2017; Pan et al., 2017; Tanvir et al., 2017).

Rapid follow-up of the gravitational wave and GRB signal in $\mathrm{X}$-rays did not show any source comparable to, or brighter than, a typical afterglow of a short GRB. Since both the gravitational data and the faintness of the prompt GRB emission suggested a jet viewed significantly off axis, this could be expected, as the afterglows from misaligned GRB jets have longer rise times than those of jets observed at small viewing angles (Van Eerten and MacFadyen, 2011). Therefore, X-ray monitoring with Swift/XRT, Chandra, and Nustar continued, and $\sim 10$ days after merger led to the detection with Chandra of a faint source $\left(L_{X} \simeq 10^{40} \mathrm{erg} \mathrm{s}^{-1}\right.$ ) (Evans et al., 2017; Margutti et al., 2017; Troja et al., 2017), whose intensity continued to rise up to $\sim 100$ days (D'Avanzo et al., 2018; Troja et al., 2020). Similarly, observations of $\mathrm{cm}$ and $\mathrm{mm}$ wavelengths at various arrays, including VLA and ALMA, failed to detect the source before $\sim 16$ days after the gravitational signal, which was interpreted as evidence that a jetted source accompanying the binary neutron star merger must be directed at a significant angle $\left(\geq 20^{\circ}\right)$ with respect to the line of sight (Alexander et al., 2017; Andreoni et al., 2017; Hallinan et al., 2017; Kim et al., 2017; Pozanenko et al., 2018).

The Fermi Large Area Telescope covered the sky region of GW170817 starting only $20 \mathrm{~min}$ after the merger and did not detect any emission in the energy range $0.1-1 \mathrm{GeV}$ to a limiting flux of $4.5 \times 10^{-10} \mathrm{erg} \mathrm{s}^{-1} \mathrm{~cm}^{-2}$ in the interval 1,153-2027 s after the merger (Ajello et al., 2018). Follow-up observation with the atmospheric Cherenkov experiment H.E.S.S. (0.3-8 TeV) from a few hours to $\sim 5$ days after merger returned no detection to a limit of a few $10^{-12} \mathrm{erg} \mathrm{s}^{-1} \mathrm{~cm}^{-2}$ (Abdalla et al., 2017). A summary of the results of the multiwavelength observing campaign within the first month of gravitational wave signal detection is reported in Abbott et al. (2017c).

While the radio and $\mathrm{X}$-ray detections are attributed to the afterglow of the short GRB, the ultraviolet, optical, and nearinfrared data are dominated by the kilonova at early epochs (with a possible contribution at $\$ 4$ days at blue wavelengths from cooling of shock-heated material around the neutron star merger, Piro and Kollmeier, 2018) and later on by the afterglow, as described in the next two sections.

\subsubsection{The Gamma-Ray Burst and Its Multiwavelength Afterglow}

The short GRB170817A, with an energy output of $\sim 10^{46} \mathrm{erg}$, was orders of magnitude dimmer than most short GRBs (Berger, 2014). Together with a viewing angle of $\sim 30$ deg estimated from the gravitational wave signal (Abbott et al., 2017a), this led to the hypothesis that the GRB was produced by a relativistic jet viewed at a comparable angle. However, the early light curve of the radio afterglow is not consistent with the behavior predicted for an offaxis collimated jet and rather suggests a quasispherical geometry, possibly with two components, a more collimated one and a nearly isotropic and mildly relativistic one, which is responsible also for producing the gamma-rays (Mooley et al., 2018a). This confirms numerous predictions whereby the shocked cloud surrounding a binary neutron star merger forms a mildly relativistic cocoon that carries an energy comparable to that of the jet and is responsible for the prompt emission and the early multiwavelength afterglow (Lazzati et al., 2017a; Lazzati et al., 2017b; Nakar and Piran, 2017; Bromberg et al., 2018; Xie et al., 2018) and is supported by detailed numerical simulations (Gottlieb et al., 2018; Lazzati et al., 2018).

Using milliarcsecond resolution radio VLBI observations at 75 and 230 days, Mooley et al. (2018b) detected superluminal motion with $\beta=3-5$, while Ghirlanda et al. (2019) determined that, at 207 days, the source is still angularly smaller than two milliarcseconds at the $90 \%$ confidence, which excludes that a nearly isotropic, mildly relativistic outflow is responsible for the radio emission, as in this case the source apparent size, after more than six months of expansion, should be significantly larger and resolved by the VLBI observation. These observations point to a structured jet as the source of GRB170817A, with a narrow opening angle $\left(\theta_{o p} \simeq 3.4\right.$ degrees $)$ and an energetic core $\left(\sim 3 \times 10^{52} \mathrm{erg}\right)$ seen under a viewing angle of $\sim 15^{\circ}$ (Ghirlanda et al., 2019). This is further confirmed by later 
radio observations, extending up to 300 days after merger, that show a sharp downturn of the radio light curve, suggestive of a jet rather than a spherical source (Mooley et al., 2018c).

The optical/near-infrared kilonova component subsided rapidly (see Section 3.1.2) leaving room to the afterglow emission: the HST observations at $\sim 100$ days after the explosion show a much brighter source than inferred from the extrapolation of the early kilonova curve to that epoch (Lyman et al., 2018). This late-epoch flux is thus not consistent with kilonova emission and is rather due to the afterglow produced within an off-axis structured jet (Fong et al., 2019). At X-ray energies, the GRB counterpart is still detected with Chandra three years after explosion (Troja et al., 2020), but its decay is not fully compatible with a structured jet, indicating that the physical conditions have changed or that an extra component is possibly emerging (e.g., a nonthermal aftermath of the kilonova ejecta; see next section).

\subsubsection{The Kilonova}

The early ground-based optical and near-infrared and spacebased (with Swift/UVOT) near-ultraviolet follow-up observations started immediately after identification of the optical counterpart of GW170817, detecting a rapid rise ( $\sim 1$ day timescale, Arcavi et al., 2017) and wavelength-dependent time decay, quicker at shorter wavelengths (Andreoni et al., 2017; Cowperthwaite et al., 2017; Díaz et al., 2017; Drout et al., 2017; Evans et al., 2017; McCully et al., 2017; Nicholl et al., 2017; Tanvir et al., 2017; Utsumi et al., 2017; Villar et al., 2017). The optical light is polarized at the very low level of $(0.50 \pm 0.07) \%$ at 1.46 days, consistent with intrinsically unpolarized emission scattered by Galactic dust, indicating that no significant alignment effect in the emission or geometric preferential direction is present in the source at this epoch, consistent with expectation for kilonova emission (Covino et al., 2017).

Starting the same night when the optical counterpart was detected, low resolution spectroscopy was carried out at the Magellan telescope (Shappee et al., 2017). This spectrum shows that the source is not yet transparent as it is emitting black body radiation, whose maximum lies however blueward of the sampled wavelength range, suggesting that the initial temperature may have been larger than $\sim 10,000 \mathrm{~K}$. The following night (1.5 days after merger) the spectrum is still described by an almost perfect black body law whose maximum at $\sim 5000 \mathrm{~K}$ was fully resolved by spectroscopy at the Very Large Telescope (VLT) with the X-Shooter spectrograph over the wavelength range 3,500-24,000 $\AA$ (Pian et al., 2017). At this epoch, the expansion velocity of the expelled ejecta, whose total mass was estimated to be 0.02-0.05 $\mathrm{M}_{\odot}$ (Pian et al., 2017; Smartt et al., 2017; Waxman et al., 2018), was $\sim 20 \%$ of the light speed, which is only mildly relativistic and therefore much less extreme than the ultrarelativistic kinematic regime of the GRB and of its early afterglow, analogous to the observed difference between the afterglows and the supernovae accompanying long GRBs. At 2.5 days after merger, the spectrum starts deviating from a black body as the ejecta become increasingly transparent and absorption lines are being imprinted on the spectral continuum by the atomic species present in the ejecta

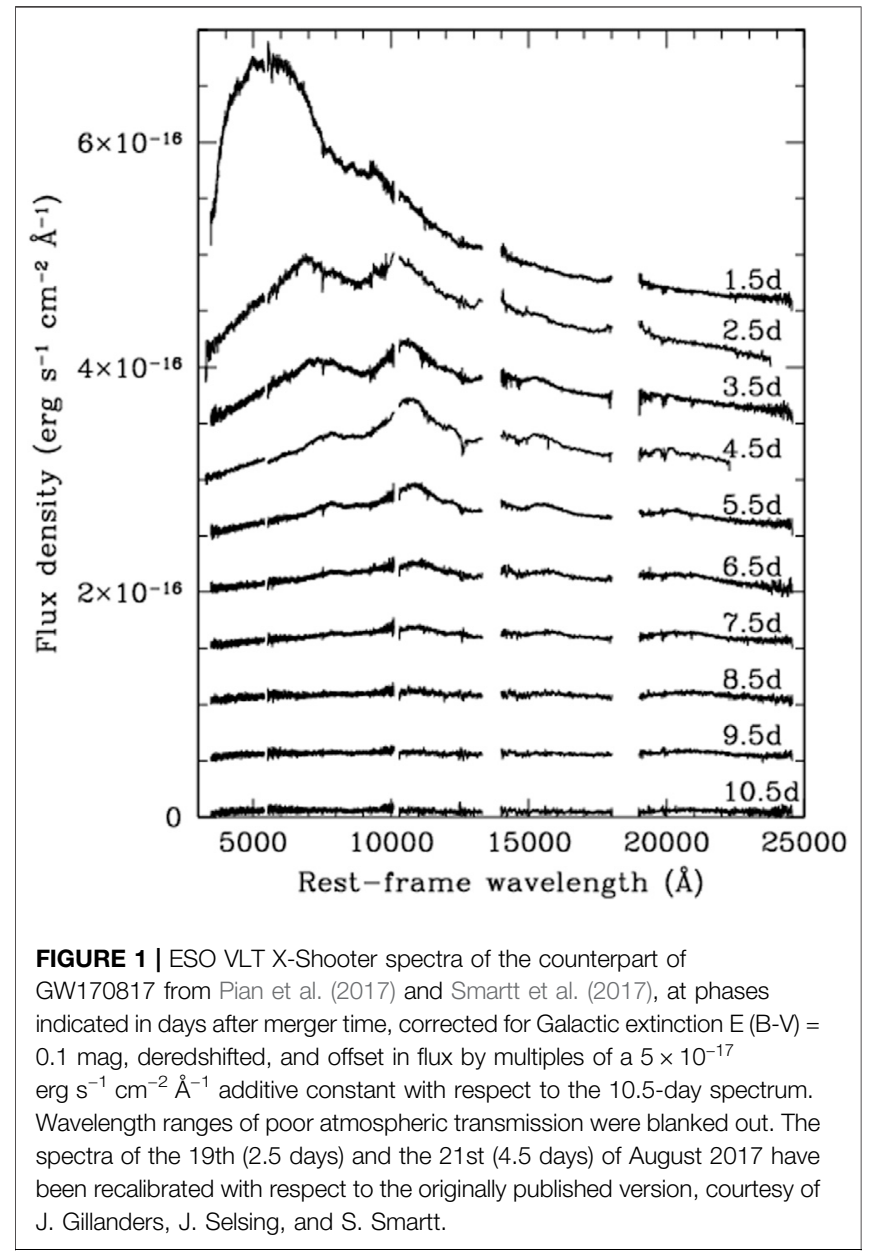

(Chornock et al., 2017; Pian et al., 2017; Smartt et al., 2017). In the following days these features become prominent and they evolve as the ejecta decelerate and the photosphere recedes (Figure 1).

In particular, in the spectrum at day 1.5 an absorption feature extending from $\sim 7,000$ to $\sim 8,100 \AA$ is detected, so that Smartt et al. (2017) preliminarily identified atomic transitions occurring in neutral $\mathrm{Cs}$ and Te, broadened and blueshifted by $\sim 0.2 c$, consistent with the expansion velocity of the photosphere. In the second spectrum (2.5 days) the Cs I and Te I lines are still detected at somewhat larger wavelengths, compatibly with a reduced photospheric expansion speed. These lines were however later disproved on account of the fact that, at the temperature of the ejecta immediately below the photosphere $(\sim 3700 \mathrm{~K})$, numerous transitions of other lanthanide elements of higher ionization potential should be observed besides Cs and Te, but are not (Watson et al., 2019). Watson et al. (2019) reanalyzed the absorption feature observed at $7,000-8,100 \AA$ and an absorption feature at $\sim 3,500 \AA$ with the aid of local thermodynamic equilibrium models with abundances from a solar-scaled r-process and from metal-poor stars and determined that the absorption features can be identified with Sr II. In the spectra at the successive epochs the line at the longer wavelength is still detected and develops a $\mathrm{P}$ Cygni profile. 
Strontium is a very abundant element and is produced close to the first r-process peak. Its possible detection makes it important to consider lighter r-process elements in addition to the lanthanides in shaping the kilonova emission spectrum (Watson et al., 2019).

At $\sim 10$ days after merger, the kilonova spectrum fades out of the reach of the largest telescopes. The radioactive source could still be monitored photometrically for another week in optical and near-infrared (Cowperthwaite et al., 2017; Drout et al., 2017; Kasliwal et al., 2017; Pian et al., 2017; Smartt et al., 2017; Tanvir et al., 2017); it was last detected at $4.5 \mu \mathrm{m}$ with the Spitzer satellite 74 days after merger (Villar et al., 2018). The kilonova ejecta are also expected to interact with the circum-binary medium and produce low-level radio and X-ray emission that peaks years after the merger (Kathirgamaraju et al., 2019). The search for this component has not returned (yet) a detection at radio wavelengths (Hajela et al., 2019), but it may start to be revealed at X-rays (Troja et al., 2020).

\subsubsection{The Host Galaxy of GW170817}

HST and Chandra images, combined with VLT MUSE integral field spectroscopy of the optical counterpart of GW170817, show that its host Galaxy, NGC 4993, is a lenticular (S0) Galaxy at $z=$ 0.009783 that has undergone a recent $(\sim 1 \mathrm{Gyr})$ galactic merger (Levan et al., 2017; Palmese et al., 2017). This merger may be responsible for igniting weak nuclear activity. No globular or young stellar cluster is detected at the location of GW170817, with a limit of a few thousand solar masses for any young system. The population in the vicinity is predominantly old and the extinction from local interstellar medium is low. Based on these data, the distance of NGC4993 was determined to be $41.0 \pm 3.1$ Mpc (Hjorth et al., 2017). The HST imaging made it also possible to establish the distance of NGC4993 through the surface brightness fluctuation method with an uncertainty of $\sim 6 \%(40.7 \pm 1.4 \pm 1.9$ $\mathrm{Mpc}$, random and systematic errors, respectively), making it the most precise distance measurement for this Galaxy (Cantiello et al., 2018). Combining this with the recession velocity measured from optical spectroscopy of the Galaxy, corrected for peculiar motions, returns a Hubble constant $H_{0}=71.9 \pm 7.1 \mathrm{~km} \mathrm{~s}^{-1} \mathrm{Mpc}^{-1}$.

Based only on the gravitational data and the standard siren argument and assuming that the optical counterpart represents the true sky location of the gravitational-wave source instead of marginalizing over a range of potential sky locations, Abbott et al. (2017d) determined a "gravitational" distance of $43.8_{-6.9}^{+2.9} \mathrm{Mpc}$ that is refined with respect to the one previously reported in Abbott et al. (2017a). Together with the corrected recession velocity of NGC4993, this yields a Hubble constant $H_{0}=70_{-8}^{+12}$ $\mathrm{km} \mathrm{s}^{-1} \mathrm{Mpc}^{-1}$, comparable to, but less precise than, that obtained from the superluminal motion of the radio counterpart core, $H_{0}=$ 70.3 $3_{-5.0}^{+5.3} \mathrm{~km} \mathrm{~s}^{-1} \mathrm{Mpc}^{-1}$ (Hotokezaka et al., 2019).

\section{KILONOVA LIGHT CURVE AND SPECTRUM}

The unstable isotopes formed during coalescence of a binary neutron star system decay radioactively and the emitted gamma-ray photons are downscattered to the ultraviolet, optical, and infrared thermal radiation that constitutes the kilonova source (Section 3.1.2). Its time decline is determined by the convolution of radioactive decay chain curves of all present unstable nuclei. This is analogous to the supernova phenomenon, where however the vastly dominant radioactive chain is ${ }^{56} \mathrm{Ni}$ decaying into ${ }^{56} \mathrm{Co}$. and then into ${ }^{56} \mathrm{Fe}$.

While radioactive nuclei decay, atoms recombine, as the source is cooling, and absorption features are imprinted in the kilonova spectra. Among neutron-rich nuclei, the lanthanides (atomic numbers 57-71) series have full f-shells and therefore numerous atomic transitions that suppress the spectrum at shorter wavelengths $(\leq 8,000 \AA)$. Spectra of dynamical ejecta of kilonova may therefore be heavily intrinsically reddened, depending on the relative abundance of lanthanides (Barnes and Kasen, 2013; Kasen et al., 2013; Tanaka and Hotokezaka, 2013). Prior to the clear detection of kilonova accompanying GW170817 (Section 3), such a source may have been detected in HST images in near-infrared $\mathrm{H}$ band of the afterglow of GRB130603B (Berger et al., 2013; Tanvir et al., 2013). Successive claims for association with short GRBs and kilonova radiation were similarly uncertain (Jin et al., 2015; Jin et al., 2016).

If the neutron stars coalescence does not produce instantaneously a black hole and a hypermassive neutron star is formed as a transitory remnant, a neutrino wind is emitted that may inhibit the formation of neutrons and reduce the amount of neutron-rich elements (Fernández and Metzger, 2013; Kajino et al., 2014; Kiuchi et al., 2014; Metzger and Fernández, 2014; Perego et al., 2014; Kasen et al., 2015; Lippuner et al., 2017). This "postmerger" kilonova component, of preferentially polar direction, is thus relatively poor in lanthanides and gives rise to a less reddened spectrum (Kasen et al., 2017; Tanaka et al., 2017).

The optical/near-infrared spectral behavior of kilonova is analogous to that of supernovae with the largest kinetic energies $\left(>10^{52} \mathrm{erg}\right.$ ), like those associated with GRBs: the large photospheric velocities broaden the absorption lines and blueshift them in the direction of the observer. Furthermore, broadening causes the lines to blend, which makes it difficult to isolate and identify individual atomic species (Iwamoto et al., 1998; Mazzali et al., 2000; Nakamura et al., 2001). While these effects can be controlled and deconvolved with the aid of a radiation transport model as it has been done for supernovae of all types (Mazzali et al., 2016; Hoeflich et al., 2017; Ergon et al., 2018; Hillier and Dessart, 2019; Ashall and Mazzali, 2020; Shingles et al., 2020), a more fundamental hurdle in modeling kilonova spectra consists in the much larger number of electronic transitions occurring in r-process element atoms than in the lighter ones that populate supernova ejecta, and in our extremely limited knowledge of individual atomic opacities of these neutron-rich elements, owing to the lack of suitable atomic data. First systematic atomic structure calculations for lanthanides and for all r-process elements were presented by Fontes et al. (2020) and Tanaka et al. (2020), respectively.

\section{SUMMARY AND FUTURE PROSPECTS}

The gravitational and electromagnetic event of August 17, 2017, provided the long-awaited confirmation that binary neutron star 
mergers are responsible for well identifiable gravitational signals at $\mathrm{kHz}$ frequencies, for short GRBs, and for thermal sources, a. k.a. kilonovae or macronovae, produced by the radioactive decay of unstable heavy elements synthesized via r-process during the coalescence. The intensive and long-term electromagnetic monitoring from ground and space allowed clear detection of the counterpart at all wavelengths. Brief $(\sim 2 \mathrm{~s})$ gamma-ray emission, peaking at $\sim 200 \mathrm{keV}$ and lagging the gravitational signal by $1.7 \mathrm{~s}$, is consistent with a weak short GRB. At ultraviolet-to-near-infrared wavelengths, the kilonova component-never before detected to this level of accuracy and robustness-dominates during the first 10 days and decays rapidly under detection threshold thereafter, while an afterglow component emerges around day $\sim 100$. Up to the most recent epochs of observation (day $\sim 1,000$ at X-rays), the kilonova does not add significantly to the bright radio and X-ray afterglow component. Multiepoch VLBI observations measured-for the first time in a GRB-superluminal motion of the radio source, thus providing evidence of late-epoch emergence of a collimated off-axis relativistic jet.

Doubtlessly, this series of breakthroughs were made possible by the closeness of the source $(40 \mathrm{Mpc})$, almost unprecedented for GRBs, and by the availability of first-class ground-based and spaceborne instruments. The many findings and exceptional new physical insight afforded by GW170817/GRB170817A make it a rosetta stone for future similar events. When a sizable group of sources with good gravitational and electromagnetic detections will be available, the properties of binary systems containing at least one neutron star, of their mergers and their aftermaths, can be mapped. It will then become possible to clarify how the dynamically ejected mass depends on the binary system parameters, mass asymmetry, and neutron stars equation of state (Ruffert and Janka, 2001; Hotokezaka et al., 2013), how the jet forms and evolves, which kinematic regimes and geometry it takes up in time, and how the GRB and afterglow observed phenomenologies can help distinguish the intrinsic properties from viewing angle effects (Janka et al., 2006; Lamb and Kobayashi, 2018; Ioka and Nakamura, 2019), what the detailed chemical content of kilonova ejecta is and how the r-process abundance pattern inferred from kilonova spectra compares with the history of heavy elements cosmic enrichment (Rosswog et al., 2018), how kilonovae can help constrain the binary neutron star rates and how the parent population of short GRBs evolves (Guetta and Stella, 2009; Yang et al., 2017; Belczynski et al., 2018; Artale et al., 2019; Matsumoto and Piran, 2020), and how gravitational and electromagnetic data can be used jointly to determine the cosmological parameters (Schutz, 1986; Del Pozzo, 2012; Abbott et al., 2017d), to mention only some fundamental open problems. Comparison of the optical and near-infrared light curves of GW170817 kilonova with those of short GRBs with known redshift suggests infact significant diversity in the kilonova component luminosities (Gompertz et al., 2018; Rossi et al., 2020).

Regrettably, short GRBs viewed at random angles, and not pole on, are relativistically beamed away from the observer direction and kilonovae are intrinsically weak. These circumstances make electromagnetic detections very difficult if the sources lie at more than $\sim 100 \mathrm{Mpc}$, as proven during the third and latest observing run (Apr 2019-Mar 2020) of the gravitational interferometers network.
In this observing period, two merger events possibly involving neutron stars were reported by the LIGO-Virgo consortium: GW190425, caused by the coalescence of two compact objects of masses each in the range 1.12-2.52 $\mathrm{M}_{\odot}$, at $\sim 160 \mathrm{Mpc}$ (Abbott et al., 2020a), and GW190814, caused by a $23 \mathrm{M}_{\odot}$ black hole merging with a compact object of $2.6 \mathrm{M}_{\odot}$ at $\sim 240 \mathrm{Mpc}$ (Abbott et al., 2020b). In neither case did the search for an optical or infrared counterpart return a positive result (Coughlin et al., 2019; Gomez et al., 2019; Ackley et al., 2020; Andreoni et al., 2020; Antier et al., 2020; Kasliwal, 2020), owing presumably to the large distance and sky error areas, although a short GRB may have been detected by the INTEGRAL SPI-ACS simultaneously with GW190425 (Pozanenko et al., 2019). Note that all coalescing stars may have been black holes, as the neutron star nature of the binary members lighter than $3 \mathrm{M}_{\odot}$ could not be confirmed.

The search for electromagnetic counterparts of gravitational radiation signals is currently thwarted primarily by the large uncertainty of their localization in the sky, which is usually no more accurate than several dozens of square degrees. Much smaller error boxes are expected to be available when the KAGRA (which had already joined LIGO-Virgo in the last months of the 2019-2020 observing run) and the INDIGO interferometers will operate at full regime as part of the network during the next observing run (Abbott et al., 2018). Observing modes, strategies, and simulations are being implemented to optimize the electromagnetic multiwavelength search and follow-up (Bartos et al., 2016; Patricelli et al., 2018; Cowperthwaite et al., 2019; Graham et al., 2019; Artale et al., 2020), and new dedicated space-based facilities are designed with critical capabilities of large sky area coverage and rapid turnaround (e.g., ULTRASAT, Sagiv et al., 2014; THESEUS, Amati et al., 2018, Stratta et al., 2018; DORADO, Cenko, 2019), to maximize the chance of detection of dim, fast-declining transients.

Finally, the possible detection of elusive $\mathrm{MeV}$ and $>\mathrm{GeV}$ neutrinos associated with the kilonova (Kyutoku and Kashiyama, 2018) and with the GRB (Bartos et al., 2019; Aartsen et al., 2020), respectively, will bring an extra carrier of information into play and thus complete the multimessenger picture associated with the binary neutron star merger phenomenon. Gravitational waves from binary neutron star inspirals and mergers; gamma-ray photons-downscattered to UV/optical/ infrared light-from radioactive decay of unstable nuclides of heavy elements, freshly formed after the merger; multiwavelength photons from nonthermal mechanisms in the relativistic jet powered by the merger remnant; and thermal and high-energy neutrinos accompanying the remnant cooling and hadronic processes in the jet, respectively, all collectively underpin the role of the four physical interactions. This fundamental role of compact star merger phenomenology thus points to the formidable opportunity offered by a multimessenger approach: bringing the communities of astrophysicists and nuclear physicists closer will foster that crossfertilization and interdisciplinary coordination that is not only beneficial but also essential for progress in this field.

\section{AUTHOR CONTRIBUTIONS}

The author confirms being the sole contributor of this work and has approved it for publication. 


\section{ACKNOWLEDGMENTS}

The author is indebted to T. Belloni, S. Cristallo, Th. Janka, P. Mazzali, A. Possenti, M. Tanaka, and F. Thielemann for discussion. She acknowledges hospitality from Liverpool John Moores University, Weizmann Institute of Science, Rehovot, and the

\section{REFERENCES}

Aartsen, M. G., Ackermann, M., Adams, J., Aguilar, J. A., Ahlers, M., Ahrens, M., et al. (2020). IceCube search for neutrinos coincident with compact binary mergers from LIGO-virgo's first gravitational-wave transient catalog. Astrophys. J. 898, L10. doi:10.3847/2041-8213/ab9d24

Abbott, B. P., Abbott, R., Abbott, T. D., Abernathy, M. R., Acernese, F., Ackley, K., et al. (2016). Properties of the binary black hole merger GW150914. Phys. Rev. Lett. 116, 241102. doi:10.1103/PhysRevLett.116.241102

Abbott, B. P., Abbott, R., Abbott, T. D., Acernese, F., Ackley, K., Adams, C., et al. (2017a). GW170817: observation of gravitational waves from a binary neutron star inspiral. Phys. Rev. Lett. 119, 161101. doi:10.1103/PhysRevLett.119.161101

Abbott, B. P., Abbott, R., Abbott, T. D., Acernese, F., Ackley, K., Adams, C., et al. (2017b). Gravitational waves and gamma-rays from a binary neutron star merger: GW170817 and GRB 170817A. Astrophys. J. 848, L13. doi:10.3847/ 2041-8213/aa920c

Abbott, B. P., Abbott, R., Abbott, T. D., Acernese, F., Ackley, K., Adams, C., et al. $(2017 \mathrm{c})$. Multi-messenger observations of a binary neutron star merger. Astrophys. J. 848, L12. doi:10.3847/2041-8213/aa91c9

Abbott, B. P., Abbott, R., Abbott, T. D., Acernese, F., Ackley, K., Adams, C., et al. (2017d). A gravitational-wave standard siren measurement of the Hubble constant. Nature 551, 85. doi:10.1038/nature 24471

Abbott, B. P., Abbott, R., Abbott, T. D., Acernese, F., Ackley, K., Adams, C., et al. (2017e). Search for post-merger gravitational waves from the remnant of the binary neutron star merger GW170817. Astrophys. J. 851, L16. doi:10.3847/ 2041-8213/aa9a35

Abbott, B. P., Abbott, R., Abbott, T. D., Abernathy, M. R., Acernese, F., Ackley, K., et al. (2018). Prospects for observing and localizing gravitational-wave transients with Advanced LIGO, Advanced Virgo and KAGRA. Living Rev. Relat. 21, 3. doi:10.1007/s41114-018-0012-9

Abbott, B. P., Abbott, R., Abbott, T. D., Abraham, S., Acernese, F., Ackley, K., et al. (2019). GWTC-1: a gravitational-wave transient catalog of compact binary mergers observed by LIGO and Virgo during the first and second observing runs. Phys. Rev. X 9, 031040. doi:10.1103/PhysRevX.9.031040

Abbott, B. P., Abbott, R., Abbott, T. D., Abraham, S., Acernese, F., Ackley, K., et al. (2020a). Observation of a compact binary coalescence with total mass $3.4 \mathrm{M}_{\odot}$. Astrophys. J. 892, L3. doi:10.3847/2041-8213/ab75f5

Abbott, B. P., Abbott, T. D., Abraham, S., Acernese, F., Ackley, K., Adams, C., et al. (2020b). GW190814: gravitational waves from the coalescence of a 23 solar mass black hole with a 2.6 solar mass compact object. Astrophys. J. 896, L44. doi:10.3847/2041-8213/ab960f

Abdalla, H., Abramowski, A., Aharonian, F., Ait Benkhali, F., Angüner, E. O., Arakawa, M., et al. (2017). TeV gamma-ray observations of the binary neutron star merger GW170817 with H. E.S.S.. Astrophys. J. 850, L22. doi:10.3847/2041$8213 /$ aa97d2

Abe, K., Bronner, C., Hayato, Y., Ikeda, M., Iyogi, K., and Kameda, J. (2018). Search for neutrinos in super-kamiokande associated with the GW170817 neutronstar merger. Astrophys. J. 857, L4. doi:10.3847/2041-8213/aabaca

Ackley, K., Amati, L., Barbieri, C., Bauer, F. E., Benetti, S., and Bernardini, M. G., (Forthcoming 2020). Observational constraints on the optical and near-infrared emission from the neutron star-black hole binary merger S190814bv. Astr. Astrophys Available at: https://arxiv.org/abs/2002.01950 (Accessed February 5, 2020).

Ajello, M., Allafort, A., Axelsson, M., Baldini, L., Barbiellini, G., Baring, M. G., et al. (2018). Fermi-LAT observations of LIGO/Virgo event GW170817. Astrophys. J. 861, 85. doi:10.3847/1538-4357/aac515
Hebrew University of Jerusalem, Israel; National Astronomical Observatory of Japan, Tokyo; Beihang University, Beijing; Yunnan National Astronomical Observatory, Kunming, China; and MaxPlanck Institute for Astrophysics and Munich Institute for Astroand Particle Physics, Garching, Germany, where part of this work was accomplished.

Albert, A., Andre, M., Anghinolfi, M., Ardid, M., Aubert, J.-J., Aublin, J., et al. (2017). Search for high-energy neutrinos from binary neutron star merger GW170817 with ANTARES, IceCube, and the Pierre auger observatory. Astrophys. J. 850, L35. doi:10.3847/2041-8213/aa9aed

Alexander, K. D., Berger, E., Fong, W., Williams, P. K. G., Guidorzi, C., Margutti, R., et al. (2017). The electromagnetic counterpart of the binary neutron star merger LIGO/Virgo GW170817. VI. Radio constraints on a relativistic jet and predictions for late-time emission from the kilonova ejecta. Astrophys. J. 848, L21. doi:10.3847/2041-8213/aa905d

Amati, L., O’Brien, P., Götz, D., Bozzo, E., Tenzer, C., Frontera, F., et al. (2018). The THESEUS space mission concept: science case, design and expected performances. Adv. Space Res. 62, 191. doi:10.1016/j.asr.2018.03.010

Andreoni, I., Ackley, K., Cooke, J., Acharyya, A., Allison, J. R., Anderson, G. E., et al. (2017). Follow up of GW170817 and its electromagnetic counterpart by Australianled observing programmes. Pub. Astron. Soc. Au. 34, 69. doi:10.1017/pasa.2017.65

Andreoni, I., Goldstein, D. A., Kasliwal, M. M., Nugent, P. E., Zhou, R., Newman, J. A., et al. (2020). GROWTH on S190814bv: deep synoptic limits on the optical/ near-infrared counterpart to a neutron star-black hole merger. Astrophys. J. 890, 131. doi:10.3847/1538-4357/ab6a1b

Annala, E., Gorda, T., Kurkela, A., and Vuorinen, A. (2018). Gravitational-wave constraints on the neutron-star-matter equation of state. Phys. Rev. Lett. 120, 172703. doi:10.1103/PhysRevLett.120.172703

Antier, S., Agayeva, S., Almualla, M., Awiphan, S., Baransky, K., Barynova, S., et al. (2020). GRANDMA observations of advanced LIGO's and advanced Virgo's third observational campaign. Mon. Not. Roy. Astron. Soc. 497, 5518. doi:10. 1093/mnras/staa1846

Antonelli, L. A., D’Avanzo, P., Perna, R., Amati, L., Covino, S., Cutini, S., et al. (2009). Grb 090426: the farthest short gamma-ray burst? Astron. Astrophys. 507, L45. doi:10.1051/0004-6361/200913062

Arcavi, I., Hosseinzadeh, G., Andrew Howell, D., McCully, C., Poznanski, D., Kasen, D., et al. (2017). Optical emission from a kilonova following a gravitational-wave-detected neutron-star merger. Nature 551, 64. doi:10. 1038/nature24291

Artale, M. C., Michela, M., Nicola, G., Sabha, N. B., Mario, S., Santoliquido, F., et al. (2019). Host galaxies of merging compact objects: mass, star formation rate, metallicity, and colours. Mon. Not. Roy. Astron. Soc. 487, 1675. doi:10.1093/ mnras/stz1382

Artale, M. C., Bouffanais, Y., Mapelli, M., Giacobbo, N., Sabha, N. B., Santoliquido, F., et al. (2020). An astrophysically motivated ranking criterion for low-latency electromagnetic follow-up of gravitational wave events. Mon. Not. Roy. Astron. Soc. 495, 1841. doi:10.1093/mnras/staa 1252

Ashall, C., and Mazzali, P. (2020). Extracting high-level information from gammaray burst supernova spectra. Mon. Not. Roy. Astron. Soc. 492, 5956. doi:10.1093/ mnras/staa212

Banerjee, P., Haxton, W. C., and Qian, Y.-Z. (2011). Long, cold, early r process? Neutrino-Induced nucleosynthesis in He shells revisited. Phys. Rev. Lett. 106, 201104. doi:10.1103/PhysRevLett.106.201104

Barnes, J., and Kasen, D. (2013). Effect of a high opacity on the light curves of radioactively powered transients from compact. object mergers. Astrophys. J. 775, 18. doi:10.1088/0004-637X/775/1/18

Bartos, I., Huard, T. L., and Márka, S. (2016). James webb space telescope can detect kilonovae in gravitational wave follow-up search. Astrophys. J. 816, 61. doi:10. 3847/0004-637X/816/2/61

Bartos, I., Veske, D., Keivani, A., Marka, Z., Countryman, S., Blaufuss, E., et al. (2019). Bayesian multimessenger search method for common sources of gravitational waves and high-energy neutrinos. Phys. Rev. D 100, 083017. doi:10.1103/PhysRevD.100.083017 
Bauswein, A., Just, O., Janka, H.-J., and Stergioulas, N. (2017). Neutron-star radius constraints from GW170817 and future detections. Astrophys. J. 850, L34. doi:10.3847/2041-8213/aa9994

Belczynski, K., Askar, A., Arca-Sedda, M., Chruslinska, M., Donnari, M., Giersz, M., et al. (2018). The origin of the first neutron star - neutron star merger. Astron. Astrophys. 615, A91. doi:10.1051/0004-6361/201732428

Beniamini, P., Duran, R. B., Petropoulou, M., and Giannios, D. (2020). Ready, set, launch: time interval between a binary neutron star merger and short gammaray burst jet formation. Astrophys. J. 895, L33. doi:10.3847/2041-8213/ab9223

Berger, E., Fong, W., and Chornock, R. (2013). An r-process kilonova associated with the short-hard GRB 130603B. Astrophys. J. 774, L23. doi:10.1088/2041$8205 / 774 / 2 / \mathrm{L} 23$

Berger, E. (2014). Short-duration gamma-ray bursts. Annu. Rev. Astron. Astrophys. 52, 43. doi:10.1146/annurev-astro-081913-035926

Blanchard, P. K., Berger, E., Fong, W., Nicholl, M., Leja, J., Conroy, C., et al. (2017). The electromagnetic counterpart of the binary neutron star merger LIGO/Virgo GW170817. VII. Properties of the host galaxy and constraints on the merger timescale. Astrophys. J. 848, L22. doi:10.3847/2041-8213/aa9055

Blanchet, L. (2014). Gravitational radiation from post-Newtonian sources and inspiralling compact binaries. Living Rev. Relat. 17, 2. doi:10.12942/lrr-2014-2

Blinnikov, S. I., Novikov, I. D., Perevodchikova, T. V., and Polnarev, A. G. (1984). Exploding neutron stars in close binaries. Sov. Astron. Lett. 10, 177.

Bromberg, O., Nakar, E., Piran, T., and Sari, R. (2018). The $\gamma$-rays that accompanied GW170817 and the observational signature of a magnetic jet breaking out of NS merger ejecta. Mon. Not. Roy. Astron. Soc. 475, 2971. doi:10. 1093/mnras/stx3316

Bromberg, O., Nakar, E., Piran, T., and Sari, R. (2013). Short versus long and collapsars versus non-collapsars: a quantitative classification of gamma-ray bursts. Astrophys. J. 764, 179. doi:10.1088/0004-637X/764/2/179

Burbidge, E. M., Burbidge, G. R., Fowler, W. A., and Hoyle, F. (1957). Synthesis of the elements in stars. Rev. Mod. Phys. 29, 547. doi:10.1103/RevModPhys.29.547

Burgay, M., D’Amico, N., Possenti, A., Manchester, R. N., Lyne, A. G., and Joshi, B. C. (2003). An increased estimate of the merger rate of double neutron stars from observations of a highly relativistic system. Nature. 426, 531. doi:10.1038/ nature 02124

Burgio, G. F., Drago, A., Pagliara, G., Schulze, H.-J., and Wei, J.-B. (2018). Are small radii of compact stars ruled out by GW170817/AT2017gfo?. Astrophys. J. 860, 139. doi:10.3847/1538-4357/aac6ee

Burns, E., Connaughton, V., Zhang, B.-B., Lien, A., Briggs, M. S., Goldstein, A., et al. (2016). Do the Fermi gamma-ray burst monitor and Swift burst alert telescope see the same short gamma-ray bursts?. Astrophys. J. 818, 110. doi:10. 3847/0004-637X/818/2/110

Busso, M., Gallino, R., and Wasserburg, G. J. (1999). Nucleosynthesis in asymptotic giant branch stars: relevance for galactic enrichment and solar system formation. Annu. Rev. Astron. Astrophys. 37, 239. doi:10.1146/annurev.astro.37.1.239

Cantiello, M., Jensen, J. B., Blakeslee, J. P., Berger, E., Levan, A. J., Tanvir, N. R., et al. (2018). A precise distance to the host galaxy of the binary neutron star merger GW170817 using surface brightness fluctuations. Astrophys. J. 854, L31. doi:10.3847/2041-8213/aaad64

Cenko, S. B. (2019). The gravitational-wave ultraviolet counterpart imager (GUCI) network. American Astronomical Society Meeting Abstracts 234, 212.

Chornock, R., Berger, E., Kasen, D., Cowperthwaite, P. S., Nicholl, M., Villar, V. A., et al. (2017). The electromagnetic counterpart of the binary neutron star merger LIGO/Virgo GW170817. IV. Detection of near-infrared signatures of r-process nucleosynthesis with gemini-south. Astrophys. J. 848, L19. doi:10.3847/2041$8213 /$ aa905c

Chruslinska, M., Belczynski, K., Klencki, J., and Benacquista, M. (2018). Double neutron stars: merger rates revisited. Mon. Not. Roy. Astron. Soc. 474, 2937. doi:10.1093/mnras/stx2923

Côté, B., Fryer, C. L., Belczynski, K., Korobkin, O., Chruślińska, M., Vassh, N., et al. (2018). The origin of r-process elements in the milky way. Astrophys. J. 855, 99. doi:10.3847/1538-4357/aaad67

Côté, B., Eichler, M., Arcones, A., Hansen, C. J., Simonetti, P., Frebel, A., et al. (2019). Neutron star mergers might not Be the only source of r-process elements in the milky way. Astrophys. J. 875, 106. doi:10.3847/1538-4357/ ab10db

Coughlin, M. W., Ahumada, T., Anand, S., De, K., Hankins, M. J., Kasliwal, M. M., et al. (2019). GROWTH on S190425z: searching thousands of square degrees to identify an optical or infrared counterpart to a binary neutron star merger with the zwicky transient facility and palomar gattini-IR. Astrophys. J. 885, L19. doi:10.3847/2041-8213/ab4ad8

Coulter, D. A., Foley, R. J., Kilpatrick, C. D., Drout, M. R., Piro, A. L., Shappee, B. J., et al. (2017). Swope Supernova Survey 2017a (SSS17a), the optical counterpart to a gravitational wave source. Science 358, 1556. doi:10.1126/science.aap9811

Covino, S., Wiersema, K., Fan, Y. Z., Toma, K., Higgins, A. B., Melandri, A., et al. (2017). The unpolarized macronova associated with the gravitational wave event GW 170817. Nature Astr 1, 791. doi:10.1038/s41550-017-0285-z

Cowan, J. J., Sneden, C., Lawler, J. E., Aprahamian, A., Weischer, M., Langanke, M., et al. (Forthcoming 2020). Origin of the heaviest elements: the rapid neutroncapture process. Rev. Mod. Phys Available at: https://arxiv.org/abs/1901.01410 (Accessed January 5, 2020).

Cowperthwaite, P. S., Berger, E., Villar, V. A., Metzger, B. D., Nicholl, M., Chornock, R., et al. (2017). The electromagnetic counterpart of the binary neutron star merger LIGO/Virgo GW170817. II. UV, optical, and near-infrared light curves and comparison to kilonova models. Astrophys. J. 848, L17. doi:10. 3847/2041-8213/aa8fc7

Cowperthwaite, P. S., Villar, V. A., Scolnic, D. M., and Berger, E. (2019). LSST target-of-opportunity observations of gravitational-wave events: essential and efficient. Astrophys. J. 874, 88. doi:10.3847/1538-4357/ab07b6

D’Avanzo, P., Campana, S., Salafia, O. S., Ghirlanda, G., Ghisellini, G., Melandri, A., et al. (2018). The evolution of the X-ray afterglow emission of GW 170817/ GRB 170817A in XMM-Newton observations. Astron. Astrophys. 613, L1. doi:10.1051/0004-6361/201832664

Davies, M. B., Benz, W., Piran, T., and Thielemann, F. K. (1994). Merging neutron stars. I. Initial results for coalescence of noncorotating systems. Astrophys. J. 431, 742. doi:10.1086/174525

Del Pozzo, W. (2012). Inference of the cosmological parameters from gravitational waves: application to second generation interferometers. Phys. Rev. D 86, 043011. doi:10.1103/PhysRevD.86.043011

Díaz, M. C., Macri, L. M., Garcia Lambas, D., Mendes de Oliveira, C., Nilo Castellon, L., Ribeiro, T., et al. (2017). Observations of the first electromagnetic counterpart to a gravitational-wave source by the TOROS collaboration. Astrophys. J. 848, L29. doi:10.3847/2041-8213/aa9060

Drout, M. R., Piro, A. L., Shappee, B. J., Kilpatrick, C. D., Simon, J. D., Contreras, C., et al. (2017). Light curves of the neutron star merger GW170817/SSS17a: implications for r-process nucleosynthesis. Science. 358, 1570. doi:10.1126/ science.aaq0049

Eichler, D., Livio, M., Piran, T., and Schramm, D. N. (1989). Nucleosynthesis, neutrino bursts and gamma-rays from coalescing neutron stars. Nature 340, 126. doi:10.1038/340126a0

Epstein, R. I., Colgate, S. A., and Haxton, W. C. (1988). Neutrino-induced r-process nucleosynthesis. Phys. Rev. Lett. 61, 2038. doi:10.1103/PhysRevLett.61.2038

Ergon, M., Fransson, C., Jerkstrand, A., Kozma, C., Kromer, M., and Spricer, K. (2018). Monte-Carlo methods for NLTE spectral synthesis of supernovae. Astron. Astrophys. 620, A156. doi:10.1051/0004-6361/201833043

Evans, P. A., Cenko, S. B., Kennea, J. A., Emrey, S. W. K., Kuin, N. P. M., Korobkin, O., et al. (2017). Swift and NuSTAR observations of GW170817: detection of a blue kilonova. Science. 358, 1565. doi:10.1126/science.aap9580

Fang, K., and Metzger, B. D. (2017). High-energy neutrinos from millisecond magnetars formed from the merger of binary neutron stars. Astrophys. J 849, 153. doi:10.3847/1538-4357/aa8b6a

Fernández, R., and Metzger, B. D. (2013). Delayed outflows from black hole accretion tori following neutron star binary coalescence. Mon. Not. Roy. Astron. Soc. 435, 502. doi:10.1093/mnras/stt1312

Fernández, R., and Metzger, B. D. (2016). Electromagnetic signatures of neutron star mergers in the advanced LIGO era. Annu. Rev. Nucl. Part Sci. 66, 23. doi:10. 1146/annurev-nucl-102115-044819

Fong, W., Berger, E., and Fox, D. B. (2010). Hubble space telescope observations of short gamma-ray burst host galaxies: morphologies, offsets, and local environments. Astrophys. J. 708, 9. doi:10.1088/0004-637X/708/1/9

Fong, W., Berger, E., Margutti, R., and Zauderer, B. A. (2015). A decade of shortduration gamma-ray burst broadband Afterglows: energetics, circumburst densities, and jet opening angles. Astrophys. J. 815, 102. doi:10.1088/0004$637 \mathrm{X} / 815 / 2 / 102$

Fong, W., Blanchard, P. K., Alexander, K. D., Strader, J., Margutti, R., Hajela, A., et al. (2019). The optical afterglow of GW170817: an off-axis structured jet and 
deep constraints on a globular cluster origin. Astrophys. J. 883, L1. doi:10.3847/ 2041-8213/ab3d9e

Fontes, C. J., Fryer, C. L., Hungerford, A. L., Wollaeger, R. T., and Korobkin, O. (2020). A line-binned treatment of opacities for the spectra and light curves from neutron star mergers. Mon. Not. Roy. Astron. Soc. 493, 4143. doi:10.1093/ mnras/staa 485

Freiburghaus, C., Rosswog, S., and Thielemann, F. (1999). R-process in neutron star mergers. Astrophys. J. 525, L121. doi:10.1086/312343

Fryer, C. L., Herwig, F., Hungerford, A., and Timmes, F. X. (2006). Supernova fallback: a possible site for the r-process. Astrophys. J. 646, L131. doi:10.1086/ 507071

Galama, T. J., Vreeswjik, P. M., van Paradijs, J., Kouveliotou, C., Augusteijn, T., and Bohnhardt, H. (1998). An unusual supernova in the error box of the $\gamma$-ray burst of 25 April 1998. Nature 395, 670. doi:10.1038/27150

Gehrels, N., Cannizzo, J. K., Kanner, J., Kasliwal, M. M., Nissanke, S., and Singer, L. P. (2016). Galaxy strategy for LIGO-virgo gravitational wave counterpart searches. Astrophys. J. 820, 136. doi:10.3847/0004-637X/820/2/136

Ghirlanda, G., Salafia, O. S., Paragi, Z., Giroletti, M., Yang, J., Marcote, B., et al. (2019). Compact radio emission indicates a structured jet was produced by a binary neutron star merger. Science. 363, 968. doi:10.1126/science.aau8815

Goldstein, A., Veres, P., Burns, E., Briggs, M. S., Hamburg, R., Kocevski, D., et al. (2017). An ordinary short gamma-ray burst with extraordinary implications: fermi-GBM detection of GRB 170817A. Astrophys. J. 848, L14. doi:10.3847/ 2041-8213/aa8f41

Gomez, S., Hosseinzadeh, G., Cowperthwaite, P. S., Villar, V. A., Berger, E., Gardner, T., et al. (2019). A galaxy-targeted search for the optical counterpart of the candidate NS-BH merger S190814bv with magellan. Astrophys. J. 884, L55. doi:10.3847/2041-8213/ab4ad5

Gompertz, B. P., Levan, A. J., Tanvir, N. R., Hjorth, J., Covino, S., Evans, P. A., et al. (2018). The diversity of kilonova emission in short gamma-ray bursts. Astrophys. J. 860, 62. doi:10.3847/1538-4357/aac206

Gottlieb, O., Nakar, E., Piran, T., and Hotokezaka, K. (2018). A cocoon shock breakout as the origin of the $\gamma$-ray emission in GW170817. Mon. Not. Roy. Astron. Soc. 479, 588. doi:10.1093/mnras/sty1462

Graham, M. J., Kulkarni, S. R., Bellm, E. C., Adams, S. M., Barbarino, C., Blagorodnova, N., et al. (2019). The zwicky transient facility: science objectives. Publ. Astron. Soc. Pac. 131, 078001. doi:10.1088/1538-3873/ab006c

Guetta, D., and Stella, L. (2009). Short $\gamma$-ray bursts and gravitational waves from dynamically formed merging binaries. Astron. Astrophys. 498, 329. doi:10.1051/ 0004-6361:200810493

Hajela, A., Margutti, R., Alexander, K. D., Kathirgamaraju, A., Baldeschi, A., Guidorzi, C., et al. (2019). Two years of nonthermal emission from the binary neutron star merger GW170817: rapid fading of the jet afterglow and first constraints on the kilonova fastest ejecta. Astrophys. J. 886, L17. doi:10.3847/ 2041-8213/ab5226

Hallinan, G., Corsi, A., Mooley, K. P., Hotokezaka, K., Nakar, E., Kasliwal, M. M., et al. (2017). A radio counterpart to a neutron star merger. Science 358, 1579. doi:10.1126/science.aap9855

Hillebrandt, W., Takahaski, K., and Kodama, T. (1976). R-process nucleosynthesis: a dynamical model. Astron. Astrophys. 52, 63.

Hillier, D. J., and Dessart, L. (2019). Photometric and spectroscopic diversity of Type II supernovae. Astron. Astrophys. 631, A8. doi:10.1051/0004-6361/ 201935100

Hjorth, J., Levan, A. J., Tanvir, N. R., Lyman, J. D., Wojtak, R., Schrøder, S. L., et al. (2017). The distance to NGC 4993: the host galaxy of the gravitational-wave event GW170817. Astrophys. J. 848, L31. doi:10.3847/2041-8213/aa9110

Hoeflich, P., Hsiao, E. Y., Ashall, C., Burns, C. R., Diamond, T. R., Phillips, M. M., et al. (2017). Light and color curve properties of type ia supernovae: theory versus observations. Astrophys. J. 846, 58. doi:10.3847/1538-4357/aa84b2

Horowitz, C. J., Arcones, A., Côté, B., Dillmann, I., Nazarewicz, W., Roederer, I. U., et al. (2019). R-process nucleosynthesis: connecting rare-isotope beam facilities with the cosmos. J. Phys. G Nucl. Part. Phys. 46, 083001. doi:10.1088/1361-6471/ab0849

Hotokezaka, K., Kuichi, K., Kyutoku, K., Okawa, H., Sekiguchi, Y.-i., Shibata, M., et al. (2013). Mass ejection from the merger of binary neutron stars. Phys. Rev. D 87, 024001. doi:10.1103/PhysRevD.87.024001

Hotokezaka, K., Nakar, E., Gottlieb, O., Nissanke, S., Masuda, K., Hallinan, G., et al. (2019). A Hubble constant measurement from superluminal motion of the jet in GW170817. Nature Astr 3, 940. doi:10.1038/s41550-019-0820-1
Hulse, R. A., and Taylor, J. H. (1974). A high-sensitivity pulsar survey. Astrophys. J. 191, L59. doi:10.1086/181548

Hulse, R. A., and Taylor, J. H. (1975). Discovery of a pulsar in a binary system. Astrophys. J. 195, L51. doi:10.1086/181708

Im, M., Yoon, Y., Lee, S. J., Lee, H. M., Kim, J., Lee, C.-U., et al. (2017). Distance and properties of NGC 4993 as the host galaxy of the gravitational-wave source GW170817. Astrophys. J. 849, L16. doi:10.3847/2041-8213/aa9367

Ioka, K., and Nakamura, T. (2019). Spectral puzzle of the off-axis gamma-ray burst in GW170817. Mon. Not. Roy. Astron. Soc 487, 4884. doi:10.1093/mnras/ stz1650

Iwamoto, K., Mazzali, P. A., Nomoto, K., Umeda, H., Nakamura, T., Patat, F., et al. (1998). A hypernova model for the supernova associated with the $\gamma$-ray burst of 25 April 1998. Nature. 395, 672. doi:10.1038/27155

Janka, H. -Th., Aloy, M.-A., Mazzali, P. A., and Pian, E. (2006). Off-Axis properties of short gamma-ray bursts. Astrophys. J. 645, 1305. doi:10.1086/504580

Ji, A. P., Frebel, A., Chiti, A., and Simon, J. D. (2016). R-process enrichment from a single event in an ancient dwarf galaxy. Nature 531, 610. doi:10.1038/ nature 17425

Jin, Z.-P., Li, X., Cano, Z., Covino, S., Fan, Y.-Z., Wei, D.-M., et al. (2015). The light curve of the macronova associated with the long-short burst GRB 060614 . Astrophys. J. 811, L22. doi:10.1088/2041-8205/811/2/L22

Jin, Z.-P., Hotokezaka, K., Li, X., Tanaka, M., D’Avanzo, P., Fan, Y.-Z., et al. (2016). The Macronova in GRB 050709 and the GRB-macronova connection. Nat. Commun. 7, 12898. doi:10.1038/ncomms12898

Jin, Z.-P., Xiang, L., Hao, W., Yuan, Z.-W., Hao, N.-H., Yuan, Q., et al. (2018). Short GRBs: opening angles, local neutron star merger rate, and off-axis events for GRB/GW association. Astrophys. J. 857, 128. doi:10.3847/1538-4357/aab76d

Kajino, T., Grant, J. M., and Takehito, H. (2014). Neutrinos in core-collapse supernovae and nucleosynthesis. J. Phys. G Nucl. Phys. 41, 044007. doi:10.1088/ 0954-3899/41/4/044007

Kajino, T., Aoki, W., Balantekin, A. B., Diehl, R., Famiano, M. A., Mathews, G J., et al. (2019). Current status of r-process nucleosynthesis. Prog. Part. Nucl. Phys. 107, 109. doi:10.1016/j.ppnp.2019.02.008

Kasen, D., Badnell, N. R., and Barnes, J. (2013). Opacities and spectra of the r-process ejecta from neutron star mergers. Astrophys. J. 774, 25. doi:10.1088/ 0004-637X/774/1/25

Kasen, D., Rodrigo, F., and Brian, D. M. (2015). Kilonova light curves from the disc wind outflows of compact object mergers. Mon. Not. Roy. Astron. Soc. 450, 1777. doi:10.1093/mnras/stv721

Kasen, D., Metzger, B., Barnes, J., Quataeart, E., and Ramirez-Ruiz, E. (2017). Origin of the heavy elements in binary neutron-star mergers from a gravitational-wave event. Nature 551, 80. doi:10.1038/nature24453

Kasliwal, M. M., Nakar, E., Singer, L. P., Kaplan, D. L., Cook, D. O., Van Sistine, A., et al. (2017). Illuminating gravitational waves: a concordant picture of photons from a neutron star merger. Science 358, 1559. doi:10.1126/science.aap9455

Kasliwal, M. M., Anand, S., Ahumada, T., Stein, R., Carracedo, A. S., Andreoni, I., et al. (Forthcoming 2020). Kilonova luminosity function constraints based on zwicky transient facility searches for 13 neutron star mergers. Astrophys. $J$ Available at: https://arxiv.org/abs/2006.11306 (Accessed June 19, 2020).

Kathirgamaraju, A., Giannios, D., and Beniamini, P. (2019). Observable features of GW170817 kilonova afterglow. Mon. Not. Roy. Astron. Soc. 487, 3914. doi:10. 1093/mnras/stz1564

Kim, S., Schulze, S., Resmi, L., González-López, J., Higgins, A. B., Ishwara-Chandra, C. H., et al. (2017). ALMA and GMRT constraints on the off-axis gamma-ray burst 170817A from the binary neutron star merger GW170817. Astrophys. J. 850, L21. doi:10.3847/2041-8213/aa970b

Kimura, S. S., Murase, K., Bartos, I., Ioka, K., Heng, I. S., and Mészáros, P. (2018). Transejecta high-energy neutrino emission from binary neutron star mergers. Phys. Rev. D 98, 043020. doi:10.1103/PhysRevD.98.043020

Kiuchi, K., Kyutoku, K., Sekiduchi, Y., Shibata, M., and Wada, T. (2014). High resolution numerical relativity simulations for the merger of binary magnetized neutron stars. Phys. Rev. D. 90, 041502. doi:10.1103/PhysRevD.90.041502

Kouveliotou, C., Meegan, C. A., Fishman, G. J., Bhat, N. P., Briggs, M. S., Koshut, T. M., et al. (1993). Identification of two classes of gamma-ray bursts. Astrophys. J. 413, L101. doi:10.1086/186969

Kyutoku, K., and Kashiyama, K. (2018). Detectability of thermal neutrinos from binary neutron-star mergers and implications for neutrino physics. Phys. Rev. D 97, 103001. doi:10.1103/PhysRevD.97.103001 
Lamb, G. P., and Kobayashi, S. (2018). GRB 170817A as a jet counterpart to gravitational wave triggerGW 170817. Mon. Not. Roy. Astron. Soc. 478, 733. doi:10.1093/mnras/sty1108

Lamb, G. P., Tanvir, N., Levan, A., Postigo, A. d. U., Kawaguchi, K., Corsi, A., et al. (2019). Short GRB 160821B: a reverse shock, a refreshed shock, and a wellsampled kilonova. Astrophys. J. 883, 48. doi:10.3847/1538-4357/ab38bb

Lattimer, J. M., and Schramm, D. N. (1974). Black-hole-neutron-star collisions. Astrophys. J. 192, L145. doi:10.1086/181612

Lattimer, J. M., and Schramm, D. N. (1976). The tidal disruption of neutron stars by black holes in close binaries. Astrophys. J. 210, 549. doi:10.1086/154860

Lazzati, D., Deich, A., Morsony, B. J., and Workman, J. C. (2017a). Off-axis emission of short gamma-ray bursts and the detectability of electromagnetic counterparts of gravitational-wave-detected binary mergers. Mon. Not. Roy. Astron. Soc. 471, 1652. doi:10.1093/mnras/stx1683

Lazzati, D., Lopez-Camara, D., Cantiello, M., Morsony, B. J., Perna, R., and Workman, J. C. (2017b). Off-axis prompt X-ray transients from the cocoon of short gamma-ray bursts. Astrophys. J. 848, L6. doi:10.3847/2041-8213/aa8f3d

Lazzati, D., Perna, R., Morsony, B. J., Lopez-Camara, D., Cantiello, M., Ciolfi, R., et al. (2018). Late time afterglow observations reveal a collimated relativistic jet in the ejecta of the binary neutron star merger GW170817. Phys. Rev. Lett. 120, 241103. doi:10.1103/PhysRevLett.120.241103

Levan, A., Crowther, P., Grijs, R. d., Langer, N., Xu, D., and Yoon, S.-C. (2016). Gamma-ray burst progenitors. Space Sci. Rev. 202, 33. doi:10.1007/s11214-0160312-x

Levan, A., Lyman, J. D., Tanvir, N. R., Hjorth, J., Mandel, I., Stanway, E. R., et al. (2017). The environment of the binary neutron star merger GW170817. Astrophys. J. 848, L28. doi:10.3847/2041-8213/aa905f

Li, L.-X., and Paczyński, B. (1998). Transient events from neutron star mergers. Astrophys. J. 507, L59. doi:10.1086/311680

Lim, Y., and Holt, J. W. (2018). Neutron star tidal deformabilities constrained by nuclear theory and experiment. Phys. Rev. Lett. 121, 062701. doi:10.1103/ PhysRevLett.121.062701

Lippuner, J., Fernandez, R., Roberts, L. F., Foucart, F., Rasen, D., Metzger, B. D., et al. (2017). Signatures of hypermassive neutron star lifetimes on r-process nucleosynthesis in the disc ejecta from neutron star mergers. Mon. Not. Roy. Astron. Soc. 472, 904. doi:10.1093/mnras/stx1987

Lipunov, V. M., Gorbovskoy, E., Kornilov, V. G., Tyurina, N., Balanutsa, P., Kuznetsov, A., et al. (2017). MASTER optical detection of the first LIGO/Virgo neutron star binary merger GW170817. Astrophys. J. 850, L1. doi:10.3847/20418213/aa92c0

Lyman, J. D., Lamb, G. P., Levan, A. J., Mandel, I., Tanvir, N. R., Kobayashi, S., et al. (2018). The optical afterglow of the short gamma-ray burst associated with GW170817. Nature Astr 2, 751. doi:10.1038/s41550-018-0511-3

Macias, P., and Ramirez-Ruiz, E. (2019). Constraining collapsar r-process models through stellar abundances. Astrophys. J. 877, L24. doi:10.3847/2041-8213/ab2049

Margutti, R., Berger, E., Fong, W., Guidorzi, C., Alexander, K. D., Metzger, B. D., et al. (2017). The electromagnetic counterpart of the binary neutron star merger LIGO/Virgo GW170817. V. Rising X-ray emission from an off-axis jet. Astrophys. J. 848, L20. doi:10.3847/2041-8213/aa9057

Martinez, J. G., Stovall, K., Freire, P. C. C., Deneva, J. S., Tauris, T. M., Ridolfi, A., et al. (2017). Pulsar J1411+2551: a low-mass double neutron star system. Astrophys. J. 851, L29. doi:10.3847/2041-8213/aa9d87

Matsumoto, T., and Piran, T. (2020). On short GRBs similar to GRB 170817A detected by Fermi-GBM. Mon. Not. Roy. Astron. Soc. 492, 4283. doi:10.1093/ mnras/staa050

Mazzali, P. A., Iwamoto, K., and Nomoto, K. (2000). A spectroscopic analysis of the energetic type ic hypernova SN 1997ef. Astrophys. J. 545, 407. doi:10.1086/ 317808

Mazzali, P. A., Sullivan, M., Pian, E., Greiner, J., and Kann, D. A. (2016). Spectrum formation in superluminous supernovae (Type I). Mon. Not. Roy. Astron. Soc. 458, 3455. doi:10.1093/mnras/stw512

McCully, C., Hiramatsu, D., Howell, D. A., Hosseinzadeh, G., Arcavi, I., Kasen, D., et al. (2017). The rapid reddening and featureless optical spectra of the optical counterpart of GW170817, at 2017gfo, during the first four days. Astrophys. J. 848, L32. doi:10.3847/2041-8213/aa9111

Mennekens, N., and Vanbeveren, D. (2014). Massive double compact object mergers: gravitational wave sources and r-process element production sites. Astron. Astrophys. 564, A134. doi:10.1051/0004-6361/201322198
Metzger, B. D., and Fernández, R. (2014). Red or blue? A potential kilonova imprint of the delay until black hole formation following a neutron star merger. Mon. Not. Roy. Astron. Soc. 441, 3444. doi:10.1093/mnras/stu802

Metzger, B. D. (2019). Kilonovae. Living Reviews in Relativity. 23, 1. doi:10.1007/ s41114-019-0024-0

Mooley, K., Nakar, E., Hotokezaka, K., Hallinan, G., Corsi, A., Frail, D. A., et al. (2018a). A mildly relativistic wide-angle outflow in the neutron-star merger event GW170817. Nature 554, 207. doi:10.1038/nature25452

Mooley, K., Deller, A. T., Gottlieb, O., Nakar, E., Hallinan, G., Bourke, S., et al. (2018b). Superluminal motion of a relativistic jet in the neutron-star merger GW170817. Nature 561, 355. doi:10.1038/s41586-018-0486-3

Mooley, K., Frail, D. A., Dobie, D., Lenc, E., Corsi, A., De, K., et al. (2018c). A strong jet signature in the late-time light curve of GW170817. Astrophys. J. 868, L11. doi:10.3847/2041-8213/aaeda7

Most, E. R., Lukas, R. W., Rezzolla, L., and Schaffner-Bielich, J. (2018). New constraints on radii and tidal deformabilities of neutron stars from GW170817. Phys. Rev. Lett. 120, 261103. doi:10.1103/PhysRevLett.120.261103

Nakamura, T., Mazzali, P. A., Nomoto, K., and Iwamoto, K. (2001). Light curve and spectral models for the hypernova SN 1998BW associated with GRB 980425. Astrophys. J. 550, 991. doi:10.1086/319784

Nakamura, K., Kajino, T., Mathews, G. J., Sato, S., and Harikae, S. (2013). A review of R-PROCESS nucleosynthesis in the collapsar jet. Int. J. Mod. Phys. E. 22, 1330022. doi:10.1142/S0218301313300221

Nakamura, K., Kajino, T., Mathews, G. J., Sato, S., and Harikae, A. (2015). r-process nucleosynthesis in the MHD+neutrino-heated collapsar jet. Astron. Astrophys. 582, A34. doi:10.1051/0004-6361/201526110

Nakano, H., Narikawa, T., Oohara, K.-I., Sakai, K., Shinkai, H.-A., Takahashi, H., et al. (2019). Comparison of various methods to extract ringdown frequency from gravitational wave data. Phys. Rev. D 99, 124032. doi:10.1103/PhysRevD.99. 124032

Nakar, E., and Piran, T. (2017). The observable signatures of GRB cocoons. Astrophys. J. 834, 28. doi:10.3847/1538-4357/834/1/28

Nakar, E. (2007). Short-hard gamma-ray bursts. Phys. Rep. 442, 166. doi:10.1016/j. physrep.2007.02.005

Nicholl, M., Berger, E., Kasen, D., Metzger, B. D., Elias, J., Briceno, C., et al. (2017). The electromagnetic counterpart of the binary neutron star merger LIGO/Virgo GW170817. III. Optical and UV spectra of a blue kilonova from fast polar ejecta. Astrophys. J. 848, L18. doi:10.3847/2041-8213/aa9029

Ning, H., Qian, Y.-Z., and Meyer, B. S. (2007). R-Process nucleosynthesis in shocked surface layers of O-Ne-Mg cores. Astrophys. J. 667, L159. doi:10.1086/ 522372

Nishimura, S., Kotake, K., Hashimoto, M.-A., Yamada, S., Nishimura, N., Fujimoto, S., et al. (2006). R-Process nucleosynthesis in magnetohydrodynamic jet explosions of core-collapse supernovae. Astrophys. J. 642, 410. doi:10.1086/500786

Nissanke, S., Holz, D. E., Hughes, S. A., Dalal, N., and Sievers, J. L. (2010). Exploring short gamma-ray bursts as gravitational-wave standard sirens. Astrophys. J. 725, 496. doi:10.1088/0004-637X/725/1/496

Özel, F., and Freire, P. (2016). Masses, radii, and the equation of state of neutron stars. Annu. Rev. Astron. Astrophys. 54, 401. doi:10.1146/annurev-astro081915-023322

Palmese, A., Hartley, W., Tarsitano, F., Conselice, C., Lahav, O., Allam, S. S., et al. (2017). Evidence for dynamically driven formation of the GW170817 neutron star binary in NGC 4993. Astrophys. J. 849, L34. doi:10.3847/2041-8213/aa9660

Pan, Y. -C., Kilpatrick, C. D., Simon, J. D., Xhakaj, E., Boutsia, K., Coulter, D.A., et al. (2017). The old host-galaxy environment of SSS17a, the first electromagnetic counterpart to a gravitational-wave source. Astrophys. J 848, L30. doi:10.3847/2041-8213/aa9116

Pandey, S. B., Hu, Y., Tirado, A. J. C., Pozanenko, A. S., Sanchez-Ramirez, R., Gorosabel, J., et al. (2019). A multiwavelength analysis of a collection of shortduration GRBs observed between 2012 and 2015. Mon. Not. Roy. Astron. Soc. 485, 5294. doi:10.1093/mnras/stz530

Patricelli, B., Stamerra, A., Razzano, M., Pian, E., and Cella, G. (2018). Searching for gamma-ray counterparts to gravitational waves from merging binary neutron stars with the Cherenkov Telescope Array. J. Cosmol. Astropart. Phys. 5, 056. doi:10.1088/1475-7516/2018/05/056

Perego, A., Rosswog, S., Cabezon, R., Korobkin, O., Kaeppeli, R., Arcones, A., et al. (2014). Neutrino-driven winds from neutron star merger remnants. Mon. Not. Roy. Astron. Soc. 443, 3134. doi:10.1093/mnras/stu1352 
Pfahl, E., Podsiadlowski, P., and Rappaport, S. (2005). Relativistic binary pulsars with black hole companions. Astrophys. J. 628, 343. doi:10.1086/430515

Pian, E., D’Avanzo, P., Benetti, S., Branchesi, M., Brocato, E., Campana, S., et al. (2017). Spectroscopic identification of r-process nucleosynthesis in a double neutron-star merger. Nature. 551, 67. doi:10.1038/nature24298

Piro, A. L., and Kollmeier, J. A. (2018). Evidence for cocoon emission from the early light curve of SSS17a. Astrophys. J. 855, 103. doi:10.3847/1538-4357/aaaab3

Pozanenko, A. S., Barkov, M. V., Minaev, P. V., Volnova, A. A., Mazaeva, E. D., Moskvitin, A. S., et al. (2018). GRB 170817A associated with GW170817: multifrequency observations and modeling of prompt gamma-ray emission. Astrophys. J. 852, L30. doi:10.3847/2041-8213/aaa2f6

Pozanenko, A. S., Minaev, P. Y., Grebenev, S. A., and Chelovekov, I. V. (2019). Observation of the second LIGO/Virgo event connected with a binary neutron star merger S190425z in the gamma-ray range. Astrophys. Lett. 45, 710. doi:10. $1134 /$ S1063773719110057

Radice, D., Perego, A., Zappa, F., and Bernuzzi, S. (2018). GW170817: joint constraint on the neutron star equation of state from multimessenger observations. Astrophys. J. 852, L29. doi:10.3847/2041-8213/aaa402

Ramirez-Ruiz, E., Trenti, M., Macleod, M., Roberts, L. F., Lee, W. H., SaladinoRosas, M. I., et al. (2015). Compact stellar binary assembly in the first nuclear star clusters and r-process synthesis in the early Universe. Astrophys. J. 802, L22. doi:10.1088/2041-8205/802/2/L22

Rauscher, T., Applegate, J. M., Cowan, J. J., and Weischer, M. (1994). Production of heavy elements in inhomogeneous cosmologies. Astrophys. J. 429, 499. doi:10. $1086 / 174339$

Renzo, M., Zapartas, E., de Mink, S. E., Gotberg, Y., Justham, S., Farmer, R. J., et al. (2019). Massive runaway and walkaway stars. A study of the kinematical imprints of the physical processes governing the evolution and explosion of their binary progenitors. Astron. Astrophys. 624, A66. doi:10.1051/0004-6361/ 201833297

Rossi, A., Stratta, G., Maiorano, E., Spighi, D., Masetti, N., Palazzi, E., et al. (2020). A comparison between short GRB afterglows and kilonova AT2017gfo: shedding light on kilonovae properties. Mon. Not. Roy. Astron. Soc. 493, 3379. doi:10.1093/mnras/staa4794

Rosswog, S., and Liebendörfer, M. (2003). High-resolution calculations of merging neutron stars - II. Neutrino emission. Mon. Not. Roy. Astron. Soc. 342, 673. doi:10.1046/j.1365-8711.2003.06579.x

Rosswog, S., Liebendorfer, M., Thielemann, F.-K., Davies, M. B., Benz, W., and Piran, T. (1999). Mass ejection in neutron star mergers. Astron. Astrophys. $341,499$.

Rosswog, S., Sollerman, J., Feindt, U., Goobar, A., Korobkin, O., Wollaeger, R., et al. (2018). The first direct double neutron star merger detection: implications for cosmic nucleosynthesis. Astron. Astrophys. 615, A132. doi:10.1051/0004-6361/ 201732117

Ruffert, M., and Janka, H. -Th. (2001). Coalescing neutron stars - a step towards physical models. III. Improved numerics and different neutron star masses and spins. Astron. Astrophys. 380, 544. doi:10.1051/0004-6361:20011453

Safarzadeh, M., Sarmento, R., and Scannapieco, E. (2019). On neutron star mergers as the source of r-process-enhanced metal-poor stars in the milky way. Astrophys. J. 876, 28. doi:10.1088/2041-8205/802/2/L22

Sagiv, I., Gal-Yam, A., Ofek, E. O., Waxman, E., Aharonson, O., Kulkarni, S. R., et al. (2014). Science with a wide-field UV transient explorer. Astrophys. J. 147, 79. doi:10.1088/0004-6256/147/4/79

Salafia, O. S., Ghisellini, G., Ghirlanda, G., and Colpi, M. (2018). Interpreting GRB170817A as a giant flare from a jet-less double neutron star merger. Astron. Astrophys. 619, A18. doi:10.1051/0004-6361/201732259

Sana, H., Mink, S. E. d., Koter, A. d., Langer, N., Evans, C. J., Geiles, M., et al. (2012). Binary interaction dominates the evolution of massive stars. Science. 337, 444. doi:10.1126/science.1223344

Savchenko, V., Ferrigno, C., Kuulkers, E., Bazzano, A., Bozzo, E., Brandt, S., et al. (2017). Integral detection of the first prompt gamma-ray signal coincident with the gravitational-wave event GW170817. Astrophys. J. 848, L15. doi:10.3847/ 2041-8213/aa8f94

Schutz, B. F. (1986). Determining the Hubble constant from gravitational wave observations. Nature 323, 310. doi:10.1038/323310a0

Shapiro, S. L., and Teukolsky, S. A. (1983). Black holes, white dwarfs, and neutron stars. The Physics of Compact Objects. doi:10.1002/9783527617661
Shappee, B. J., Simon, J. D., Drout, M. R., Piro, A. L., Morrell, N., Prieto, J. L., et al. (2017). Early spectra of the gravitational wave source GW170817: evolution of a neutron star merger. Science 358, 1574. doi:10.1126/science.aaq0186

Shibagaki, S., Kajino, T., Mathews, G. J., Chiba, S., Nishimura, S., Lorusso, G., et al. (2016). Relative contributions of the weak, main, and fission-recycling r-process. Astrophys. J. 816, 79. doi:10.3847/0004-637X/816/2/79

Shibata, M., and Hotokezaka, K. (2019). Neutron star collisions and the r-process. Annu. Rev. Nucl. Part Sci. 69, 41. doi:10.1146/annurev-nucl-101918-023625

Shingles, L. J., Sim, S. A., Kromer, M., Maguire, K., Bulla, M., Collins, C., et al. (2020). Monte Carlo radiative transfer for the nebular phase of Type Ia supernovae. Mon. Not. Roy. Astron. Soc. 492, 2029. doi:10.1093/mnras/stz3412

Siegel, D. M., Barnes, J., and Metzger, B. D. (2019). Collapsars as a major source of r-process elements. Nature. 569, 241. doi:10.1038/s41586-019-1136-0

Simonetti, P., Matteucci, F., Greggio, L., and Cescutti, G. (2019). A new delay time distribution for merging neutron stars tested against Galactic and cosmic data. Mon. Not. Roy. Astron. Soc. 486, 2896. doi:10.1093/mnras/stz991

Smartt, S. J., Chen, T.-W., Jerkstrand, A., Coughlin, M., Kankare, E., Sim, S. A., et al. (2017). A kilonova as the electromagnetic counterpart to a gravitational-wave source. Nature. 551, 75. doi:10.1038/nature24303

Sneden, C., Cowan, J. J., and Gallino, R. (2008). Neutron-capture elements in the early galaxy. Annu. Rev. Astron. Astrophys. 46, 241. doi:10.1146/annurev.astro. 46.060407.145207

Soares-Santos, M., Holz, D. E., Annis, J., Chornock, R., Herner, K., Berger, E., et al. (2017). The electromagnetic counterpart of the binary neutron star merger LIGO/Virgo GW170817. I. Discovery of the optical counterpart using the dark energy camera. Astrophys. J. 848, L16. doi:10.3847/2041-8213/aa9059

Stratta, G., Ciolfi, R., Amati, L., Bozzo, E., Ghirlanda, G., Maiorano, E., et al. (2018). Theseus: a key space mission concept for Multi-Messenger Astrophysics. Adv. Space Res. 62, 662. doi:10.1016/j.asr.2018.04.013

Symbalisty, E., and Schramm, D. N. (1982). Neutron star collisions and the r-process. Astrophys. Lett. 22, 143.

Takahashi, K., Witti, J., and Janka, H.-T. (1994). Nucleosynthesis in neutrinodriven winds from protoneutron stars II. The r-process. Astron. Astrophys. 286, 857.

Tanaka, M., and Hotokezaka, K. (2013). Radiative transfer simulations of neutron star merger ejecta. Astrophys. J. 775, 113. doi:10.1088/0004-637X/775/2/113

Tanaka, M., Utsumi, Y., Mazzali, P. A., Tominaga, N., Yoshida, M., Sekiguchi, Y., et al. (2017). Kilonova from post-merger ejecta as an optical and near-Infrared counterpart of GW170817. Publ. Astron. Soc. Jpn. 69, 102. doi:10.1093/pasj/psx121

Tanaka, M., Kato, D., Gaigalas, G., Rynkun, P., Radziute, L., Wanajo, S., et al. (2018). Properties of kilonovae from dynamical and post-merger ejecta of neutron star mergers. Astrophys. J. 852, 109. doi:10.3847/1538-4357/aaa0cb

Tanaka, M., Kato, D., Gaigalas, G., and Kawaguchi, K. (2020). Systematic opacity calculations for kilonovae. Mon. Not. Roy. Astron. Soc. 496, 1369. doi:10.1093/ mnras/staa1576

Tanaka, M. (2016). Kilonova/Macronova emission from compact binary mergers. Advances in Astronomy, 634197. doi:10.1155/2016/6341974

Tanvir, N. R., Levan, A. J., Fruchter, A. S., Hjorth, J., Hounsell, R. A., Weirsema, K., et al. (2013). A "kilonova" associated with the short-duration $\gamma$-ray burst GRB 130603B. Nature. 500, 547. doi:10.1038/nature 12505

Tanvir, N. R., Levan, A. J., Gonzalez-Fernandez, C., Korobkin, O., Mandel, I., Rosswog, S., et al. (2017). The emergence of a lanthanide-rich kilonova following the merger of two neutron stars. Astrophys. J. 848, L27. doi:10. 3847/2041-8213/aa90b6

Tauris, M. T., Kramer, M., Freire, P. C. C., Wex, N., Janka, H.-T., Langer, N., et al. (2017). Formation of double neutron star systems. Astrophys. J. 846, 170. doi:10. 3847/1538-4357/aa7e89

Taylor, J. H., and Weisberg, J. M. (1982). A new test of general relativity gravitational radiation and the binary pulsar PSR 1913+16. Astrophys. J. 253, 908. doi:10.1086/159690

Thielemann, F.-K., Arcones, A., Kappeli, R., Liebendorfer, M., Rauscher, T., Winteler, C., et al. (2011). What are the astrophysical sites for the r-process and the production of heavy elements? Prog. Part. Nucl. Phys. 66, 346. doi:10. 1016/j.ppnp.2011.01.032

Thielemann, F.-K., Eichler, M., Panov, I. V., and Wehmeyer, B. (2017). Neutron star mergers and nucleosynthesis of heavy elements. Annu. Rev. Nucl. Part Sci. 67, 253. doi:10.1146/annurev-nucl-101916-123246 
Tominaga, N., Tanaka, M., Morokuma, T., Utsumi, Y., Yamaguchi, M. S., Yasuda, N., et al. (2018). Subaru hyper suprime-cam survey for an optical counterpart of GW170817. Publ. Astron. Soc. Jpn. 70, 28. doi:10.1093/pasj/psy007

Troja, E., Piro, L., van Eerten, H., Wollaeger, R. T., Im, M., Fox, O. D., et al. (2017). The X-ray counterpart to the gravitational-wave event GW170817. Nature 551, 71. doi:10.1038/nature 24290

Troja, E., van Eerten, H., Zhang, B., Ryan, G., Piro, L., Ricci, R., et al. (Forthcoming 2020). A thousand days after the merger: continued X-ray emission from GW170817. Mon. Not. Roy. Astron. Soc Available at: https://arxiv.org/abs/2006. 01150 (Accessed June 1, 2020).

Utsumi, Y., Tanaka, M., Tominaga, N., Yoshida, M., Barway, S., Nagayama, T., et al. (2017). J-GEM observations of an electromagnetic counterpart to the neutron star merger GW170817. Publ. Astron. Soc. Jpn. 69, 101. doi:10.1093/pasj/psx118

Valenti, S., David, J. S., Yang, S., Cappellaro, E., Tartaglia, L., Corsi, A., et al. (2017). The discovery of the electromagnetic counterpart of GW170817: kilonova at 2017gfo/DLT17ck. Astrophys. J. 848, L24. doi:10.3847/2041-8213/aa8edf

van Eerten, H. J., and MacFadyen, A. I. (2011). Synthetic off-axis light curves for lowenergy gamma-ray bursts. Astrophys. J. 733, L37. doi:10.1088/2041-8205/733/2/L37

Villar, V. A., Guillochon, J., Berger, E., Metzger, B. D., Cowperthwaite, P. S., Nicholl, M., et al. (2017). The combined ultraviolet, optical, and near-infrared light curves of the kilonova associated with the binary neutron star merger GW170817: unified data set, analytic models, and physical implications. Astrophys. J. 851, L21. doi:10.3847/2041-8213/aa9c84

Villar, V. A., Cowperthwaite, P. S., Berger, E., Blanchard, P. K., Gomez, S., Alexander, K. D., et al. (2018). Spitzer space telescope infrared observations of the binary neutron star merger GW170817. Astrophys. J. 862, L11. doi:10. 3847/2041-8213/aad281

Wallner, A., Faestermann, T., Feige, J., Feldstein, C., Knie, K., Korschinek, G., et al. (2015). Abundance of live Pu in deep-sea reservoirs on Earth points to rarity of actinide nucleosynthesis. Nat. Commun. 6, 5956. doi:10.1086/ncomms6956

Wanajo, S., Itoh, N., Ishimaru, Y., Nozawa, S., and Beers, T. C. (2002). The r-process in the neutrino winds of core-collapse supernovae and $\mathrm{U}$-Th cosmochronology. Astrophys. J. 577, 853. doi:10.1086/342230

Wang, X.-G., Zhang, B., Liang, E.-W., Lu, R.-J., Lin, D.-B., Li, J., et al. (2018). Gamma-ray burst jet breaks revisited. Astrophys. J. 859, 160. doi:10.3847/15384357/aabc13

Watson, D., Hansen, C. J., Selsing, J., Koch, A., Malesani, D. B., Anderson, A. C., et al. (2019). Identification of strontium in the merger of two neutron stars. Nature 574, 497. doi:10.1038/s41586-019-1676-3

Waxman, E., Ofek, E. O., Doron, K., and Avishay, G.-Y. (2018). Constraints on the ejecta of the GW170817 neutron star merger from its electromagnetic emission. Mon. Not. Roy. Astron. Soc. 481, 3423. doi:10.1093/mnras/sty2441
Weisberg, J. M., and Huang, Y. (2016). Relativistic measurements from timing the binary pulsar PSR B1913+16. Astrophys. J. 829, 55. doi:10.3847/0004-637X/829/ $1 / 55$

Wheeler, J. C., Cowan, J. J., and Hillebrandt, W. (1998). The r-process in collapsing O/Ne/Mg cores. Astrophys. J. 493, L101. doi:10.1086/311133

Winteler, C., Kappeli, R., Perego, A., Arcones, A., Vasset, N., Nishimura, N., et al. (2012). Magnetorotationally driven supernovae as the origin of early galaxy r-process elements? Astrophys. J. 750, L22. doi:10.1088/2041-8205/750/1/L22

Wollaeger, R. T., Korobkin, O., Fontes, C. J., Rosswog, S. K., Even, W. P., Fryer, C. L., et al. (2018). Impact of ejecta morphology and composition on the electromagnetic signatures of neutron star mergers. Mon. Not. Roy. Astron. Soc. 478, 3298. doi:10.1093/mnras/sty1018

Wolszczan, A. (1991). A nearby 37.9-ms radio pulsar in a relativistic binary system. Nature 350, 688. doi:10.1038/350688a0

Woosley, S. E., and Bloom, J. S. (2006). The supernova gamma-ray burst connection. Annu. Rev. Astron. Astrophys. 44, 507. doi:10.1146/annurev. astro.43.072103.150558

Woosley, S. E., Wilson, J. R., Mathews, G. J., Hoffman, R. D., and Meyer, B. S. (1994). The r-process and neutrino-heated supernova ejecta. Astrophys. J. 433, 229. doi: $10.1086 / 174638$

Xie, X., Zrake, J., and MacFadyen, A. (2018). Numerical simulations of the jet dynamics and synchrotron radiation of binary neutron star merger event GW170817/GRB 170817A. Astrophys. J. 863, 58. doi:10.3847/1538-4357/aacf9c

Yang, S., Valenti, S., Cappellaro, E., Sand, D. J., Tartaglia, L., Corsi, A., et al. (2017). An empirical limit on the kilonova rate from the DLT40 one day cadence supernova survey. Astrophys. J. 851, L48. doi:10.3847/2041-8213/aaa07d

Yaron, O., and Gal-Yam, A. (2012). WISeREP - an interactive supernova data repository. Publ. Astr. Soc. Pacific 124, 668. doi:10.1086/666656

Ye, C. S., Fong, W.-F., Kremer, K., Rodriguez, C. L., Chatterjee, S., Fragione, G., et al. (2020). On the rate of neutron star binary mergers from globular clusters. Astrophys. J. 888, L10. doi:10.3847/2041-8213/ab5dc5

Conflict of Interest: The author declares that the research was conducted in the absence of any commercial or financial relationships that could be construed as a potential conflict of interest.

Copyright (c) 2021 Pian. This is an open-access article distributed under the terms of the Creative Commons Attribution License (CC BY). The use, distribution or reproduction in other forums is permitted, provided the original author(s) and the copyright owner(s) are credited and that the original publication in this journal is cited, in accordance with accepted academic practice. No use, distribution or reproduction is permitted which does not comply with these terms. 